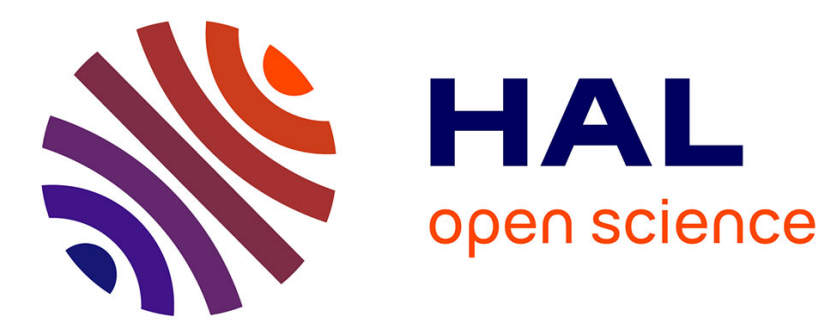

\title{
Group transformation for phase space fluids
}

\author{
J.R. Burgan, J. Guttierez, A. Munier, E. Fijalkow, M.R. Feix
}

\section{To cite this version:}

J.R. Burgan, J. Guttierez, A. Munier, E. Fijalkow, M.R. Feix. Group transformation for phase space fluids. [Research Report] Note technique CRPE n 47, Centre de recherches en physique de l'environnement terrestre et planétaire (CRPE). 1977, 50 p. hal-02191403

\section{HAL Id: hal-02191403 \\ https://hal-lara.archives-ouvertes.fr/hal-02191403}

Submitted on 23 Jul 2019

HAL is a multi-disciplinary open access archive for the deposit and dissemination of scientific research documents, whether they are published or not. The documents may come from teaching and research institutions in France or abroad, or from public or private research centers.
L'archive ouverte pluridisciplinaire HAL, est destinée au dépôt et à la diffusion de documents scientifiques de niveau recherche, publiés ou non, émanant des établissements d'enseignement et de recherche français ou étrangers, des laboratoires publics ou privés. 


\section{CENTRE DE RECHERCHE EN PHYSIQUE DE}

\section{L'ENVIRONNEMENT TERRESTRE ET PLANETAIRE}

\section{NOTE TECHNIQUE CRPE/ 47}

GROUP TRANSFORMATION FOR PHASE SPACE FLUIDS

par

J.R. BURGAN, J. GUTIERREZ, A. MUNIER, E. FIJALKOW, M.R. FEIX

$$
\text { C.R.P.E./P.C.E. }
$$

45045 - ORLEANS CEDEX

Le Chef du Département P.C.E.

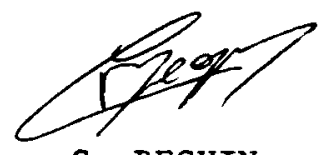

C. BEGHIN

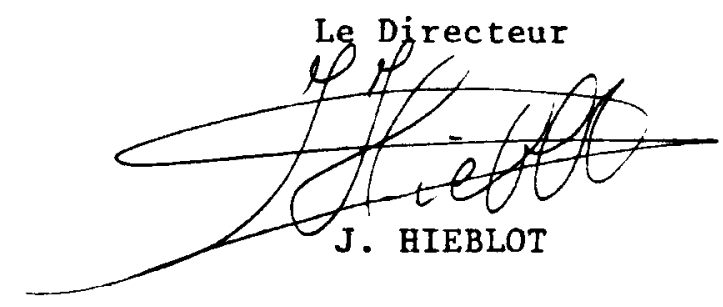


GROUP TRANSFORMATION FOR PHASE SPACE FLUIDS

J.R. BURGAN, J. GUTIERREZ*, A. MUNIER, E. FIJALKOW, M.R. FEIX

CRPE/CNRS - UNIVERSITE D'ORLEANS

INTRODUCTION

We want to study the time behaviour of systems where long distances forces are predominant. Such is the case of plasmas, accelerator beam (where we are dealing with Coulomb forces plus electromagnetic confinning external fields) and self gravitating gas (galaxy, cluster of stars etc...) where the Newtonian attraction compete against the thermal (ballistic) expansion. In many cases we can disregard the small irregularities due to the grain structure of the matter (with grain as big as a star in a galaxy) and describe the interaction through a continuous field obtained by the solution of the Poisson equation. This is the well known Vlasov Poisson structure where the global description is obtained by considering the distribution function $\mathrm{f}(\overrightarrow{\mathrm{x}}, \overrightarrow{\mathrm{v}}, \mathrm{t})$ in the 6 dimensional phase space in contradiction to a regular gas where we can usually deal with the first moments of $f$ with respect to $\vec{v}$ (particles density, momentum, energy density etc...). Consequently we call phase space fluids such systems. A discussion of the relative properties of these a priori very different fluids is given in $|1|$ and $|2|$. From the model's maker point of view adopted here they present great similarities.

The non linear solution of the Vlasov Poisson systems are of course very difficult to obtain analytically and most of the studies resort to numerical simulation. As soon as we introduce numerical algorithms the problem is raised of the correction of the long time behaviour. From our experience with differential equations this problem is best solved if we can find asymptotic series based on a systematic study of the different terms. As a matter of fact we begin to mix both analytical and numerical method and find in this case possibility of following some systems during thousands of period. 
Our group is developping ideas along this line and preliminary encouraging results are exposed in $|3||4|$.

Another "cornerstone" upon which this paper is built is the existence of singular solutions of the equations. As indicated by their names singular solutions are to be opposed to the regular (usual) solutions. Singular solutions have a special ordinarily simple strucutre which is preserved during the motion. The problem is to know if these singular solutions are never representative of the others (which at the beginning do not possess the special structure) or on the contrary are very representative of the regular solutions which could for example go asymptotically to the singular solutions. There questions are of course pratically unanswered. In plasma physics the BGK structures $|5|$ provides examples of such singular solutions. They are periodic steady state eventually moving at a constant velocity and self supporting. Their stabilities is a very complex problem $|1|$ and they, can be "transient asymptotic" solution as shown by numerical experiments $|6|$.

Steady state structures are usually not too difficult to obtain (although their number and their variety is a puzzle in plasma physics). Solutions involving the time are still more difficult. Recently the group technique has been used both in plasma physics (but on fluid models of non linear waves rather that on Vlasov-Poisson system) and on models which represent one dimensional equivalent of Navier Stakes fluid systems (Burger's, Kortweig De Vries equations etc...) Although in there cases self similar group techniques solve the problem it is fair to mention that usually the problem had been already solved. Such is the case of the famous solitons structures $|8||9|$. Incidently the method must be traced back to Boltzmann who use it to solve the heat diffusion equation.

Some confusion exists about the usefulness of the group method and the way the method should work. Usually we check the symetry of the equations with respect to continuous transformation group. We subsequently, use this symmetry to reduced by one the number of variables. Numerical solutions are easier and sometimes analytical solutions are possible. But of course since we have eliminated from the very beginning 
one degree of freedom cannot say how these solutions are affected by a modification of the initial (or boundaries) conditions.

We will consider the self similar transformations of the phase space and more precisely we will consider the transformation $|1| \quad\left\{\begin{array}{l}\vec{\xi}=\vec{x}(t / T)^{-\alpha} \\ \vec{\eta}=\vec{v}(t / T)^{1-\alpha}\end{array}\right.$

which will be justified later on. The meaning of the transformation is such that the $\vec{x}, \vec{v}, t$ dependance can be condensed into a $\vec{\xi}, \vec{n}$ transformation where $\vec{\xi}$ and $\vec{n}$ are the rescaled coordinates of the phase space and describe motion so simple that the time behaviour can be consequently taken into account by this rescaling. In (1) $T$ is an arbitrary time and it is convenient to consider that the initial time is not $t=0$ but $t=T$. From the following consideration it is clear that we are going simply to obtain generalisation of the BGK (steady state) structures.

In fact an interesting new point of view is to introduce both a rescaling like (1) and keep a time variation through the introduction of a new also rescaled time. In fact we will introduce the following transformations

$|2| \quad\left\{\begin{array}{l}\theta(t) \\ \vec{\xi}=A(t) \vec{x} \\ \vec{\eta}=B(t) \vec{x}+C(t) \vec{V}\end{array}\right.$

with a proper choice of $\theta(t), A(t), B(t)$ and $C(t)$ under a slightiy different form. This transformation has an old story Introduced by $|10|$ it has been rediscovered by R.H. Lewis $|11|$. Our group has pointed out some of the interesting properties both from theoretical and pratical point of view $|3||12|$. Now it appears under a new aspect and we will learn how to use the freedom left, (2) being really a continuous Lie group of transformation, to solve some problems. 
Now we end up this lengtly introduction with the title of the different sections. In I we will review quickly some of the results of the classical self similar group theory applied to the Vlasov Poisson system (with the absorption of the time variation in the rescaling of the new phase space). In II we will introduce the complete time phase space rescaling and will show the mathematical group structures. In III we will apply it to a very intriguing problem of cosmological theory, the structure of a self gravitating system when the gravitational constant $G$ varies with time, and we will show that the Dirac hypothesis with $G$ varying inversely with the age of the universe corresponds to a very simple roblem. In IV it will be shown that the problem of the motion of a charged particle in a uniform in space, time varying magnetic field, is very similar to the preceedings. In $V$ we will generalise the group transformation introduced in III to the quantum case and in VI we will come back to the V1asov Poisson system for plasmas (we will study non linear oscillations for plane cylindrical and spherical geometry) self gravitating gas and the problem of the expansion of a beasi under the space charge forces. 


\section{I - SELF SIMILAR GROUP}

We consider a one dimensional collisionless phase space fluid described by the Vlasov Poisson system. This fluid may be an electronic plasma (where for simplicity we suppose a motionless ion background of density $\mathrm{N}_{0}$ ) or a one species population beam. The two cases are respectively labelled $P$ and $B$. The gravitational case labelled $G$ is identical to the $B$ case with a change of sign in the Poisson 1 aw. Consequently we have three independent variables $\vec{x}, \vec{v}, t$ and two fonctions $f(\vec{x}, \vec{v}, t)$ the phase space distribution and $E(\vec{x}, t)$ the electric field connected by the following systems of equations $|3|\left\{\begin{array}{l}\frac{\partial f}{\partial t}+v \frac{\partial f}{\partial x}+E \frac{\partial f}{\partial v}=0 \\ \frac{\partial E}{\partial x}=\int_{-\infty}^{\infty} f(x, v, t) d v-N_{0}\end{array}\right.$

For simplicity we took $\mathrm{e}=\mathrm{m}=\varepsilon_{0}=1$ As we mentionned already we introduce the transformation group $E=a^{\beta} \cdot t ; \bar{x}=a^{r} x ; \bar{v}=a^{\delta} v ; \bar{E}=a^{\varepsilon} E ; \bar{f}=a^{\omega} f$

To get the formal invariance of the system we must consider the four following invariants.

$$
|4| \quad \xi=x\left(\frac{t}{T}\right)^{-\alpha} ; \eta=v\left(\frac{t}{T}\right)^{1-\alpha} ; F=f\left(\frac{t}{T}\right)^{\alpha+1} ; \varepsilon=E\left(\frac{t}{T}\right)^{2-\alpha}
$$

in (4) $\alpha$ is a real arbitrary number. $T$ is a characteristic time and it is useful to consider the time origin at $t=T$. At this time

$$
\begin{aligned}
& \xi=x ; \eta=V ; \quad F=f ; \quad \varepsilon=E . \\
& |5|\left\{\begin{array}{l}
\text { We obtain after substitution for the vlasov Poisson system } \\
\eta \frac{\partial F}{\partial \xi}+\varepsilon \frac{\partial F}{\partial \eta}-\frac{1}{T}\left\{(\alpha-1) \eta \frac{\partial F}{\partial \eta}+\alpha \xi \frac{\partial F}{\partial \xi}+(\alpha+1) F\right\}=0 \\
\frac{\partial \varepsilon}{\partial \xi}=\int_{-\infty}^{\infty} F d \eta-N_{0}\left(\frac{t}{T}\right)^{2}
\end{array}\right.
\end{aligned}
$$


From (5) we see that the transformation is possible in the $B$ case but not in the plasma case because the variable $t$ is still present in (5). Nevertheless a solution can be found in the following way, we translate the time origin at $T$ writing $t=T+\tau$. Assuming $T \rightarrow \infty$ and keeping $\tau$ finite (otherwise as large as we like) we see that $N_{0}(t / T)^{2} \rightarrow$ No.Moreover to avoid the trivial time independant solution in (5) we must let $\alpha \rightarrow \infty$ with $\alpha / T=\beta$. The transformation (4) becomes

|6| $\xi=x \exp -\beta \tau ; \eta=V \exp -\beta \tau ; F=f \exp \beta \sigma ; \varepsilon=E \exp -\beta \tau$.

and the Vlasov Poisson system becomes

$|>|\left\{\begin{array}{l}\eta \frac{\partial F}{\partial \xi}+\varepsilon \frac{\partial F}{\partial \eta}=\beta\left(F+\xi \frac{\partial F}{\partial \xi}+\eta \frac{\partial F}{\partial \eta}\right) \\ \frac{\partial \varepsilon}{\partial \xi}=\int_{-\infty}^{\infty} F d \eta-N_{0}\end{array}\right.$

Now equations (5) and (7) should be discussed and f possible solved in the $B$ and $P$ case. This has been partly done in $|19|$ and $|20|$. An interesting solution is obtained taking $\alpha=-1$ for the $B$ case. Assuming that the self consistant field has the form

$|8| \quad \varepsilon=2 \xi T^{-2}$

we get for the solution of $F$

$$
F=\psi(\eta \pi / 2-\xi)
$$

This is the "phase space stick"structure discussed in $\mid 2 d$ and generalised for a plasma in $|13|$. We see immediately that unphysical boundaries conditions appear at $\xi= \pm \infty$ where particles whith infinite velocity are allowed to appear. It has been shown in $|13|$ that these difficulties can be partly overcame through the concept of contamination which is based on the fact that particles on the extremities do not contribute to the field (as long as symetry is conserved). In fact this concept is going to be generalised and reiatroduced in III and we will not discuss it any longer for the moment. 


\section{II - A USEFUL GROUP OF TRANSFORMATION}

\section{A) Derivation of the transformation}

Let us consider the transformations given by (2) where $\overrightarrow{\mathrm{x}}$ and $\overrightarrow{\mathrm{v}}$ are the coordinate and velocity of a particle. We have

$$
\vec{V}=\vec{\eta} / C(t)-B(t) /(A(t) \cdot C(t)) \cdot \vec{\xi}
$$

We impose on the transformation two conditions

1) The phase space element should be conserved i.e.

$$
d \vec{\xi} d \vec{\eta}=d \vec{x} d \vec{v}
$$

implying that the Jacobian of the transformation must be unity ; this imposes

$$
|9| \quad A(t) C(t)=1
$$

computing $\mathrm{d} \overrightarrow{\mathrm{v}} / \mathrm{dt}$ and taking (9) into account.

$$
|10| \frac{d \vec{v}}{d t}=\frac{1}{C} \frac{d \theta}{d t} \frac{d \vec{\eta}}{d \theta}-\frac{\vec{\eta}}{c^{2}} \frac{d C}{d t}-B(t) \frac{d \theta}{d t} \frac{d \vec{\xi}}{d \theta}-\frac{d B}{d t} \vec{\xi}
$$

2) We want to keep the Hamiltonian formalism, Consequently $d \vec{\xi} / d \theta=\vec{\eta}$ and the new force $\vec{\varepsilon}=d \vec{\eta} / d \theta$ must be a function of $\vec{\xi}$ and $\theta$ only. Consequently, in the expression of $d \vec{\eta} / d \theta$ the friction term. i.e. the sum of the second and third terms in the left handside of (10) must be zero.

$111 \mid \frac{d c}{d t} \cdot \frac{1}{c^{2}}+B(t) \frac{d \theta}{d t}=0$ on the other band from a direct derivation of $\eta$ in (2)

$$
|12| \frac{d \vec{\eta}}{d \theta}=\frac{d t}{d \theta}\left\{B \frac{d \vec{x}}{d t}+\vec{x} \frac{d B}{d t}+\frac{d C}{d t} \vec{V}+C \frac{d \vec{V}}{d t}\right\}
$$


$\overrightarrow{\mathrm{x}}$ and $\overrightarrow{\mathrm{v}}$ being canonically conjugate we must again equal to zero $\left(B+\frac{d C}{d t}\right) \vec{v}$ and consequently $B=-d C / d t$. From this last relation and (11) we deduce

$$
\frac{d \theta}{d t}=\frac{1}{c^{2}}
$$

which together with (9) defines completely the transformation characterised by the arbitrary function $C(t)$. The "new field" $\vec{\varepsilon}$ acting on the particle in the new phase space, new time system is given by (12)

$$
\vec{\varepsilon}=\frac{d \vec{\eta}}{d \theta}=C^{3} \frac{d \vec{V}}{d t}-C^{3} \frac{d^{2} C}{d t^{2}} \vec{\xi}=C^{3} \vec{E}-C^{3} \frac{d^{2} C}{d t^{2}} \vec{\xi}
$$

Sometimes we will need the divergence of $\vec{\varepsilon}$ for Coulomb forces i.e. forces for which

$|15| \quad \frac{\partial \vec{E}}{\partial \vec{x}}=\sum_{i} q_{i} \delta\left(\vec{x}-\vec{x}_{i}\right)$

From (14), $\vec{x}=\vec{\xi} C$ and the relations

$$
\delta(c[\vec{\xi}-\vec{\xi}])= \begin{cases}c^{-1} \delta\left(\vec{\xi}-\vec{\xi}_{i}\right) & \text { in one dimension } \\ c^{-2} \delta\left(\vec{\xi}-\vec{\xi}_{i}\right) & \text { in two dimensions } \\ c^{-3} \delta\left(\vec{\xi}-\vec{\xi}_{i}\right) & \text { in three dimensions }\end{cases}
$$

we obtain

$|16| \frac{\partial \vec{\varepsilon}}{\partial \vec{\xi}}= \pm C^{4-d} \sum_{i} \delta\left(\vec{\xi}-\vec{\xi}_{i}\right)-d C^{3} \frac{d^{2} C}{d t^{2}}$

in the $B$ and $G$ cases with $d$ the dimensionality of the system ( $d=1,2,3$ respectively for one, two, three dimensional systems) The case of an electronic plasma with a continuous ion neutralizing background of density No introduces a supplementary term 
$|17| \frac{\partial \vec{\varepsilon}}{\partial \vec{\xi}}=c^{4-d} \sum_{i} \delta(\vec{\xi}-\vec{\xi})-C^{4} N_{0}-\alpha C^{3} \frac{d^{2} C}{d t^{2}}$

We will use extensively Eqs. (16) and (17). In the Vlasov description $\sum_{i} \delta\left(\vec{\xi}-\vec{\xi}_{i}\right)$ is replaced by $\int_{-\infty}^{\infty} \vec{F}(\vec{\xi}, \vec{\eta} \cdot \theta) d \vec{\eta} \quad$ The fact that $d \vec{\xi} d \vec{\eta}=d \vec{x} d \vec{v}$ shows that $f(\vec{x}, \vec{J}, t)=F(\vec{\xi}, \vec{\eta}, \theta)$ and the Hamltonian equations $\vec{\eta}=d \vec{\xi} / d \theta ; \vec{\varepsilon}=d \vec{\eta} / d \theta$ means that in the new phase space (with the new time) we keep invariant the vlasov equation

$|18| \quad \frac{\partial F}{\partial \theta}+\vec{\eta} \cdot \frac{\partial F}{\partial \vec{\xi}}+\vec{E} \cdot \frac{\partial F}{\partial \vec{\eta}}=0$

B) Group structure of the transformation

Each element of the transformation is characterised by the function $C(t)$ which transforms $\vec{x}, \vec{v}, t$ in $\vec{\xi}, \vec{\eta}, \theta$. We can reiterate with another transformation characterised now by $D(\theta)$ which transforms $\vec{\xi} \vec{n} \theta$ in $\vec{\lambda} \vec{\mu} \tau$. For a dimensional system introuucing the unit matrix of rank d,i.e. I. We have for the product of the two transformations, for the new time,

$$
d \tau=D^{-2} d \theta=D^{-2} C^{-2} d t=(D C)^{-2} d t
$$

For the new phase space the matrix transforming directly $\vec{x}$, $\vec{v}$ in $\vec{\lambda}, \vec{\mu}$ written.

$|20|\left(\begin{array}{cc}D^{-1} I & 0 \\ -\frac{d D}{d \theta} I & D I\end{array}\right)\left(\begin{array}{cc}C^{-1} I & 0 \\ -\frac{d C}{d t} I & C I\end{array}\right)=\left(\begin{array}{cc}C^{-1} D^{-1} I & 0 \\ \left(-C^{-1} \frac{d D}{d \theta}-D \frac{d C}{d t}\right) I & C D I\end{array}\right)=\left(\begin{array}{cc}C^{-1} D^{-1} I & 0 \\ -\frac{d}{d t}(C D) I & C D I\end{array}\right)$

The "new field" $\rightarrow$ is given by

|21| $\overrightarrow{q_{F}}=D^{3} \vec{\varepsilon}-D^{3} \frac{d^{2} D}{d \theta^{2}} \vec{\lambda}=D^{3} c^{3} \vec{E}-D^{3} c^{3} \frac{d^{2} c}{d t^{2}}-D^{3} \frac{d^{2} D}{d \theta^{2}} \vec{\lambda}$

But $\vec{\xi}=\mathrm{D} \vec{\lambda}$ and the two last terms of (21) can be written

$$
-D^{3} C^{3}\left(D \frac{d^{2} C}{d t^{2}}+\frac{1}{c^{3}} \frac{d^{2} D}{d \theta^{2}}\right) \vec{\lambda}
$$


Taking into account $\frac{d D}{d \theta}=C^{2} \frac{d D}{d t}$ and $\frac{d^{2} D}{d \theta^{2}}=C^{4} \frac{d^{2} D}{d t^{2}}+2 C^{3} \frac{d C}{d t} \cdot \frac{d D}{d t}$ (22) can be written

$$
-D^{3} C^{3}\left(D \frac{d^{2} C}{d t^{2}}+2 \frac{d C}{d t} \cdot \frac{d D}{d t}+C \frac{d^{2} D}{d t^{2}}\right)=-D^{3} C^{3} \frac{d^{2}}{d t^{2}}(D C)
$$

From (19) (20) (21) and (23) we see that the successive applications characterised by $C(t)$ and $D(\theta)$ are equivalent to the application characterised by $\mathrm{CD}$. This demonstrates the abelian group structure of the transformation. Moreover being characterised by an arbitrary function $C(t)$ the group is continuous.

\section{C) Time and Forces renormalisation}

In the new time, new phase space the motion equations are invariants and we can use all the mathematical models developped up to now the only change will be in the computation of the force through Poisson law from (16) or (17). The new field is now the sum of three fields.

- The self consistent field of the system given through Poisson low is computed in the same way except a multiplying factor $\mathrm{c}^{4-\mathrm{d}}$ which varies with time. by $\mathrm{C}^{4}$.

- In the $\mathrm{P}$ case the background ion field is multiplied

- Moreover we must introduce a transformation field given by $-c^{3}\left(d^{2} d / d t^{2}\right) \xi$

On the other hand the new time is given by

$$
\theta=\int_{0}^{t} \frac{d t^{\prime}}{d^{2}\left(t^{\prime}\right)}
$$

and we see that the time is rescaled. Since we want study the long time behaviour of the system we can choose $c(t)$ such that $\theta$ varies very slowly with $t$. In fact we can select $C(t)$ in such a way that $\theta$ goes to a limiting value $\theta_{t}$ when $t \rightarrow \infty$. We consequently proceeds to 
a renormalization of the time. This implies $C(t) \rightarrow \infty$ with $t$ and, of course, this time renormalization is obtained at the expense of a force increase. We show that in some physical problems we can select $C(t)$ to renormalize, i.e. have a new time going to a finite limit, or at least sl ow down considerably the time without introcucing infinities in the forces.

\section{III - GRAVITATIONAL SYSTEM WITH TIME VARYING GRAVITATIONAL CONSTANT}

We have seen that one consequence of the transformation was to introduce a function $C(t)$ which,elevated at a proper power, scales the force. If a physical constant describing the interaction decreases with time $C(t)$ can be selected as time increasing with a balance between the two effects while $\theta$ may go to a finite limit. In fact,for the Dirac hypothesis, the problem of the dynamical evolution is very much simplified as if the Dirac law was an example invented to show theiriterestof the group method. The Dirac hypothesis $|14||15|$ states that the gravitational constant varies with time accordingly

$$
G(t)=G_{0}(T / t)
$$

where $t$ is the age of the universe. We prefer to shift the time and consider the present time as origin. Then replacing $t$ by $t+T$ with $T=\Omega^{-1}$

$$
G(t)=G_{0} \frac{1}{1+\Omega t}
$$

From (16) considering a 3D system we can consider that in the new phase space the field is affected by the factor $G(t) C(t)$. Taking $G(t) C(t)=$ Go gives $C(t)=1+\Omega t$ and consequently the transformation field vanishes But the new time $\theta$ is now.

$$
\theta=\frac{t}{1+\Omega t}
$$

and goes to the finite limit $\Omega^{-1}$ when $t \rightarrow \infty$. Consequently the study for all time of a $\mathrm{N}$ body gravitating problem in the Dirac's hypothesis 
is identical to the study of a $\mathrm{N}$ body gravitating system where $\mathrm{G}$ remains constant during a finite time interval equal to $\Omega^{-1}$. We have been able to renormalize the time without introducing infinities in the force due to the time decreases of $G$. The Dirac law is the only one which allows to get rid of the variation of $G$ without introducing a transformation field. We have two possibilities : either we stick to the $\vec{x}, \vec{v}, t$ space and introduce (25) or we consider the $\vec{\xi}, \vec{n}, \theta$ space and consider $\bar{G}$ as strictly constant.

We will, of course, refrain from claiming that one space is more physical than the other. We must simply point out that $\theta$ is the ephemeres time computed with the hypothesis of constant G. This time should be compared with the time given by an atomic clock,i.e. a time connected to the motion of electrons governed by electrostatic forces. The comparison of the two times was made by Van Flandern. Although it is very difficult get rid of the different corrections, this last author claims a residual difference between the two times supporting the Dirac's ideas with an $\Omega^{-1}=10^{10}$ years.

Coming back to the more technical problems of computing orbits when $G$ varies with time, we consider the motion of mass point in 3 dimensions attracted by the origin with a gravitational constant given by

$$
G(t)=G_{0} \frac{1}{(1+\Omega t)^{\alpha}}
$$

We select $C(t)$ to be able to deduce the asymptotic properties and more precisely

- To avoid increasingin time transformation field and $G(\theta)$

- Within this last constraint to compress the time as much as possible with, if possible, a limiting $\theta_{\ell}$ Table 1 gives the choice of $C(t)$, the expression in the new variables $\theta, \vec{\xi}$, of the transformation field, of the gravitational constant and,finally, the relation between $\theta$ and $t$. 


\section{TABLE 1}

\begin{tabular}{|c|c|c|c|c|}
\hline$\alpha$ & $C(t)$ & $\overline{\mathbf{G}}(\theta)$ & transf. field & $\dot{\theta}(t)$ \\
\hline $0<\alpha<\frac{1}{2}$ & $(1+\Omega) t)^{\infty}$ & $\mathbf{G}_{\mathbf{0}}$ & $\frac{\alpha(1-\alpha) \Omega^{2}}{[1+(1-2 \alpha) \Omega \theta]^{2}} \vec{\xi}$ & $\Omega \theta=\frac{(1+\Omega t)^{1-2 \alpha}-1}{1-2 \alpha}$ \\
\hline$\alpha=\frac{1}{2}$ & $(1+\Omega t)^{\frac{1}{2}}$ & $\mathbf{G}_{0}$ & $\left.\left(\Omega^{2} / 4\right)\right\}$ & $\Omega 2 \theta=\log (1+\Omega t)$ \\
\hline$\frac{1}{2}<\alpha<1$ & $(1+\Omega t)^{\frac{1}{2}}$ & G. $\exp -\left(\alpha-\frac{1}{2}\right) \Omega \theta$ & $\left(\Omega^{2} / 4\right) \vec{\xi}$ & $\Omega \theta=\log (1+\Omega t)$ \\
\hline$\alpha=1$ & $1+\Omega t$ & $G_{0}$ & 0 & \multirow{2}{*}{$\begin{array}{c}\Omega \theta=\Omega t /(1+\Omega t) \\
\text { if } t \rightarrow \infty \\
\Omega \theta \rightarrow 1\end{array}$} \\
\hline$\alpha>1$ & $1+\Omega t$ & $G_{0}(1-\Omega \theta)^{\alpha-1}$ & 0 & \\
\hline
\end{tabular}

For $\alpha \geqslant 1$ the transformation field is zero, $G(\theta)$ is constant or goes to zero and $\theta \rightarrow \theta_{l}$. As a consequence $\xi$ goes to a limiting value $\xi_{\ell}$, and the asymptotic trajectory is $\vec{x}=\vec{\xi}_{\ell} \quad(1+\Omega t)$ with $\vec{x}$ increasing proportionally to the time.

In the case $1 / 2<\alpha<1$ the gravitational constant decreases exponentially with $\theta$ and the dominant field is $\Omega 2 / 4 \vec{\xi}$. The asymptotic solution for $\vec{\xi}$ is consequently.

$$
\vec{\xi}=K \exp \frac{\Omega \theta}{2}
$$

Combining with $\vec{x}=c \vec{\xi}$ the selected value of $C(t)$ in this case and coming back to the time $t$, we find that again $\vec{x}$ increases as $\Omega t$. Consequently for $\alpha>1 / 2$ the asymptotic state corresponds to freely going particles with a uniform velocity. This is not surprising since $G(t) \rightarrow 0$ when $t \rightarrow \infty$, but this last condition is not sufficient. Indeed for $\alpha \leqslant 1 / 2$ we will see that another type- of trajectory is possible. $G(\theta)$ being a constant and the repulsive transformation field going to zero for $\theta \rightarrow \infty$, we can predict two types of trajectories. For the open one the gravitational field goes to zero as $\xi^{-2}$, while the transformation field goes to zero as $\xi / \theta^{2}$, and in the case of $\xi$ varying at least as $\theta$ the transformation field, although going to zero is still dominant. 
The asymptotic time evolution is given by solving

$$
\dot{\xi}=\frac{\alpha(1-\alpha)}{(1-2 \alpha)^{2} \theta^{2}} \vec{\xi}
$$

Seeking a srolution of (29) of the form $\vec{\xi}=\vec{A} \theta$ we obtain

$$
\text { Since for } \alpha<1 / 2, \beta>1 \text { the domination of the }
$$

transformation field is enhanced. Now introducing $\vec{x}=C \vec{\xi}=\vec{A} C \theta \beta$ and expressing $\theta$ as a function of $t$ we obtain again an asymptotic solution in $t$ for $\vec{x}$.

But, of course, the initial conditions of the problem can be such that in the $\vec{\xi}$ plane the trajectories are of closed type The transformation field decreasing with $\theta$ the asymptotic trajectory is an ellipse in $\xi$ and altough $G(t) \rightarrow 0$ the particle is still influenced by the center of attraction, turning around it with a distance going to infinity as $(1+\Omega t)^{\alpha}$.

\section{IV - MOTION OF A CHARGED PARTICLE IN A UNIFORM IN SPACE, TIME}

\section{VARYING MAGNETIC FIELD}

If a time varying $G(t)$ is a simple hypothesis, the problem of a time varying magnetic field is certainly important from a practical point of view. Let as assume a uniform in space time varying magnetic field, $\vec{B}(t)=B(t) \vec{e}$, where $\vec{e}$ is a unit vector. Now a time varying $\vec{B}$ implies an electric field $\vec{E}=-\partial \vec{A} / \partial t$. Since the vector potential is

$$
\vec{A}=\frac{1}{2} \vec{B} \wedge \vec{x}
$$

we deduce

$$
|30| \quad \vec{E}=\frac{\dot{B}(t)}{2} \vec{x} \wedge \vec{e}
$$

(30) must be handled with care. The electric field depends obviously of the point of origin and is zero on the line parallel to $\vec{E}$ and passing through that origin. A priori $\vec{B}(t)$ being uniform itseems possible to take any point as the origin. The paradox is raised if we consider more carefully how the uniform magnetic field can be created. 
Here we consider a solenoid of length $L$ and redius $R$ where both $L$ and $R$ go to infinity. This configuration imposes a cylindrical symetry in the induced field and the axis of the solenoid is naturally the line where $\vec{E}=0$. From

we obtain

$$
\left\{\begin{array}{l}
\vec{\xi}=C^{-1} \vec{x} \\
\vec{\eta}=C \vec{v}-(d C / d t) \vec{x} \\
\frac{d \theta}{d t}=C^{-2} \\
\vec{F}=\vec{E}+\vec{v} \wedge \vec{B}
\end{array}\right.
$$

$|32| \quad \frac{d \vec{\eta}}{d \theta}=C^{3} \vec{E}+\eta \wedge C^{2} \vec{B}+C^{3}(d C / d t) \vec{\xi} \wedge \vec{B}-C^{3}\left(d^{2} C / d t^{2}\right) \vec{\xi}$

Introducing (30) we see that

$$
C^{3} \vec{E}+C^{3}(d C / d t) \vec{\xi} \wedge \vec{B}=C^{3}\left[\frac{1}{2} \frac{d B}{d t} C+B \frac{d C}{d t}\right] \vec{\xi} \wedge \vec{e}
$$

If we take $C(t)$ in such a way that $B(t) C^{2}(t)=B_{0}$

(33) cancels and we obtain

$|34| \quad \frac{d \vec{\eta}}{d \theta}=\eta \wedge \vec{B}_{0}-C^{3}\left(d^{2} d / d t^{2}\right) \vec{\xi}$

and we have to treat the motion of a particle in a constant magnetic field $\vec{B}$ o with the usual transformation field. Note that now $C(t)$ is imposed by the relation

$$
B(t) C^{2}(t)=B_{0}
$$

We investigate more precisely the case where

$$
B(t)=\frac{B_{0}}{(1+\Omega t)^{2 \alpha}}
$$

and we 1 ist on table II the value of the transformation field and the relation between $\theta$ and $t$. [In all cones the new magnetic field is Bo and $\left.C(t)=(1+\Omega t)^{\alpha}\right]$. 


\begin{tabular}{|c|c|c|c|}
\hline$\alpha$ & transformation field & $\Omega \theta(t)$ & $\Omega \theta_{\text {lim }}$ \\
\hline $0<\alpha<\frac{1}{2}$ & $\frac{\alpha(1-\alpha) \Omega^{2}}{[1+(1-2 \alpha) \Omega \theta]^{2}} \vec{\zeta}$ & $\frac{(1+\Omega t)^{1-2 \alpha}-1}{1-2 \alpha}$ & $\infty$ \\
\hline$\alpha=\frac{1}{2}$ & $\left.\left(\Omega^{2} / 4\right)\right\}$ & $\log (1+\Omega 2 t)$ & $\log _{\infty} \infty$ \\
\hline$\frac{1}{2}<\alpha<1$ & $\frac{\alpha(1-\alpha) \Omega^{2}}{[1-(2 \alpha-1) \Omega \theta]^{2}} \xi \begin{array}{l}\text { repulsive } \\
\text { force }\end{array}$ & $\frac{1}{2 a-1}\left\{1-\frac{1}{\left.(1+\Omega t)^{2 \alpha-1}\right\}}\right.$ & $\frac{1}{2 \alpha-1}$ \\
\hline$\alpha=1$ & (1) & $t / 1+\Omega t$ & 1 \\
\hline $1<\alpha$ & $\frac{-\alpha(\alpha-1) \Omega^{2}}{[1-(2 \alpha-1) \Omega \theta]^{2}} \vec{l} \begin{array}{l}\text { attractive } \\
\text { force }\end{array}$ & $\frac{1}{2 \alpha-1}\left\{1-\frac{1}{(1+\Omega t)^{2 e-1}}\right\}$ & $\frac{1}{2 \alpha-1}$ \\
\hline
\end{tabular}

In the new phase space for $\alpha<1 / 2$ the transformation field goes to zero and the asymptotic trajectory is a circle (since $B=B o$ ). Consequently, although the magnetic field goes to zero, the particle will always feel its presence.

The case $\alpha=1 \% / 2$ is quite interesting,(34) is written

$$
\frac{d^{2} \vec{\xi}}{d \theta^{2}}=\frac{d \vec{\xi}}{d \theta} \wedge \vec{B}_{0}+\frac{\Omega^{2}}{4} \vec{\xi}
$$

Decoupling the motion in a first one perpendicular to the magnetic field (characterised by $\xi_{1}$ and $\xi_{2}$ ) and another parallel (this last one is a trivial displacement at uniform velocity) we get the eigenfrequencies of the perpendicular motion $\omega^{2}$

$$
\left(\omega^{2}+\frac{\Omega^{2}}{4}\right)^{2}-\omega^{2} B_{0}^{2}=0
$$

Solutions of (38) are $2 \omega= \pm\left[B_{0} \pm \sqrt{B_{0}^{2}-\Omega^{2}}\right]$. We must remember that due to the choice of units $e=m=1$. Bo stands for the cyclotron frequency $(\mathrm{e} / \mathrm{m})$ Bo. We find two possibilities. 
$\left.1^{\circ}\right) B_{0}>\Omega$ all the eigenvalues are real and the particle undergoes oscillations in the $\vec{\xi}$ plane. In the $\vec{x}$ plane $x$ increase as $\sqrt{1+\Omega t}$

$\left.2^{\circ}\right) \mathrm{B}_{0}<\Omega$ two of the eigenvalues are complex and one corresponds to a growing instability in $\vec{\xi}$. The particle has an asymptotic motion closer to the free motion. For $B_{0}=0$ we recover of course the ballistic motion.

The asymptotic solutions for the case $1 / 2<\alpha<1$ is obtained noticing that the force is repulsive and that for $\theta \rightarrow \theta_{p}$ the motion is dominated by the transformation field

$$
\frac{\alpha(1-\alpha) \Omega^{2} \theta_{l}^{2}}{\left(\theta_{l}-\theta\right)^{2}} \vec{\xi}=\frac{d^{2} \vec{\xi}}{d \theta^{2}}
$$

We seek a solution of the form $\vec{\xi}=\vec{A}\left(\theta_{l}-\theta\right)^{\beta}$ and obtain $\beta=(\alpha-1) /(2 \alpha-1)$ Taking into account the relation between $\theta$ and $t$, this gives an asymptotic solution for $\vec{x}=C \vec{\xi}$ of the form $x \propto \Omega t$ indicating that the firal: motion of the particle is a ballistic free motion.

The last case $\alpha>1$ implies an attractive force. If we suppose that the transformation field is still dominant (we will check a posteriori) we get the same equation (39) and the same value for $\beta$ (but now $\beta$ is positive and the particle falls on the origin). The transformation field decreases 1ike $\left(\theta_{l}-\theta\right)^{\frac{\alpha-1}{2 \alpha-1}-2}=\left(\theta_{l}-\theta\right)^{\frac{1-3 \alpha}{2 \alpha-1}}$ while the magietic force decreases as the velocity i.e. $\left(\theta_{l}-\theta\right) \frac{\alpha-1}{2 \alpha-1}-1$ The ratio of the two forces (transformation/magnetic) varies as $\left(\theta_{p}-\theta\right)^{-1}$ and indeed for $\theta \rightarrow \theta_{\ell}$ the transformation field is the dominant one, $\xi \rightarrow 0$ as $\left(\theta_{l}-\theta\right)^{(\alpha-1) /(2 \alpha-1)}$ and $\mathrm{x}=\mathrm{C} \xi$ varies as $\Omega t$, implying in the regular $\vec{x}$ space a ballistic motion for large $t$.

\section{Figures 1 to 5 illustrates this different concept.}

Figure 1,2 and 3 shows what happens when $\alpha=.45$. In al1 cases $\Omega=1$. For $B_{0}=10$ the particles are nicely trapped in the $\vec{\xi}$ plane indicating an expanding spiral motion in $\vec{x}$. Notice that for $B o=0.1$ 


$$
00
$$




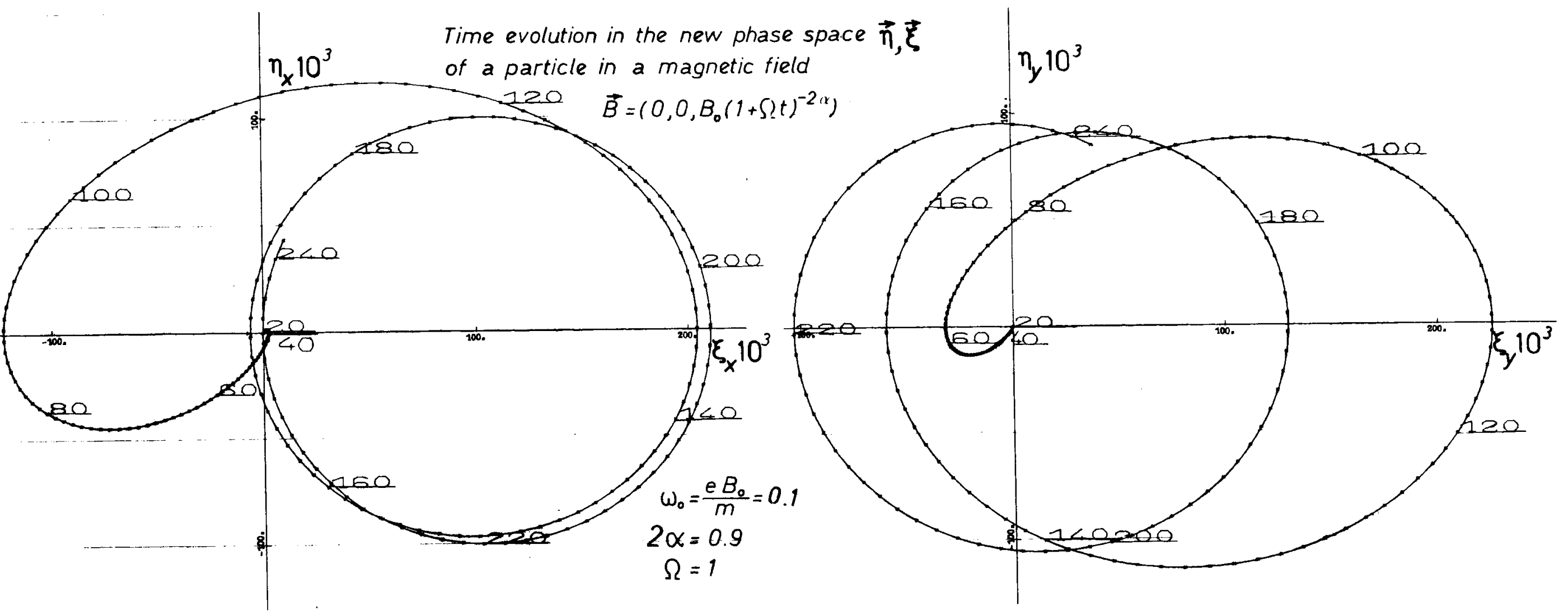

Fig. 2 


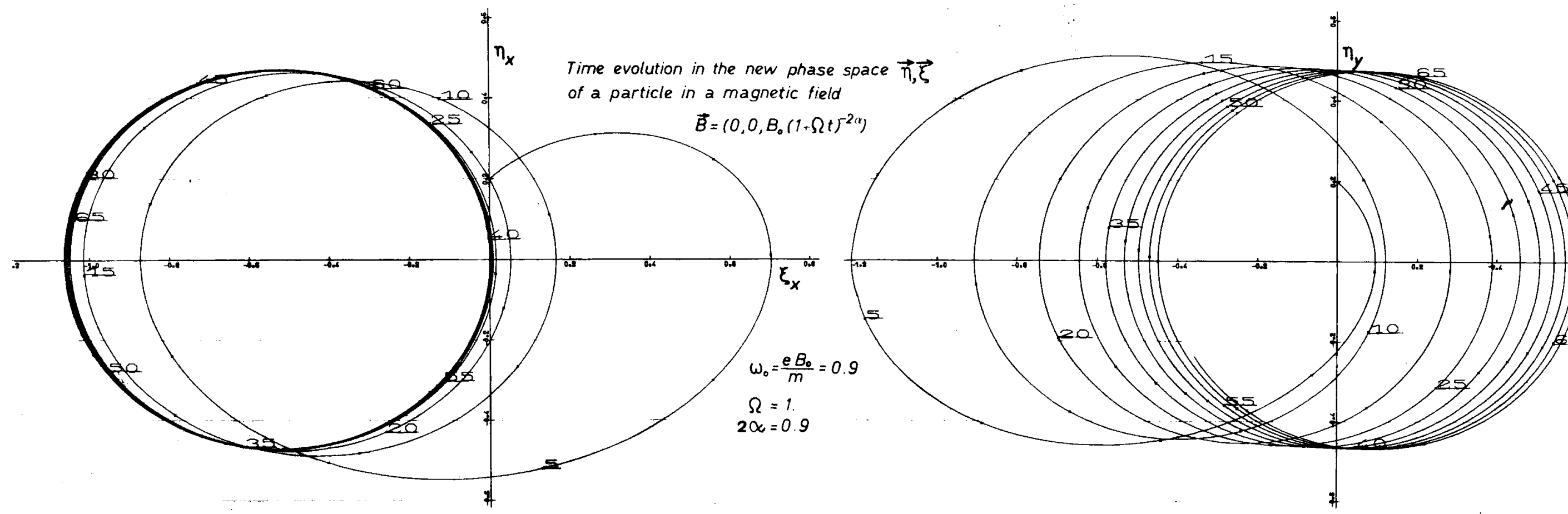

Fig. 3 


$$
00
$$




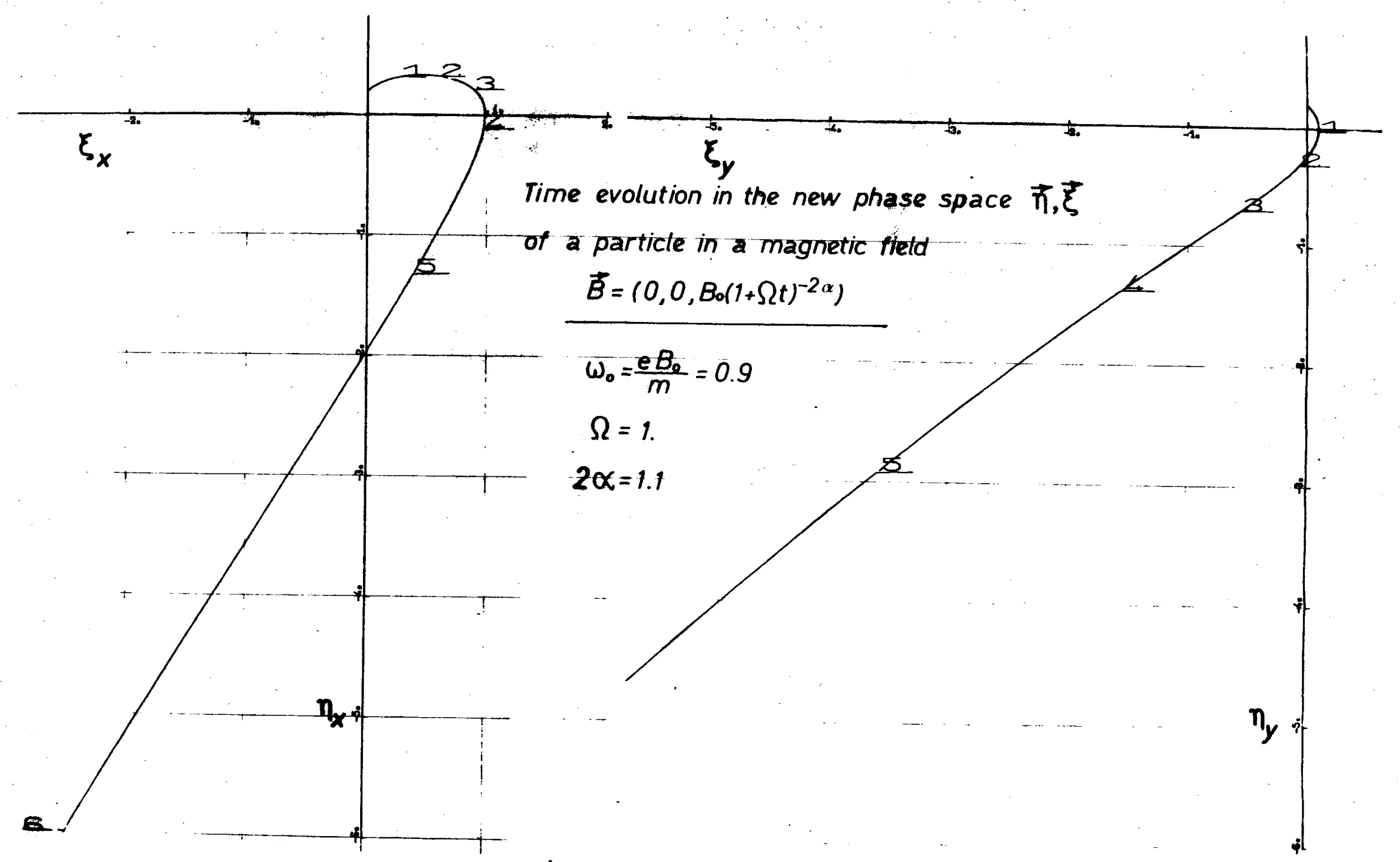

Fig. 5 
the particle gets on its circular orbit around $\theta=140$. Such a value corresponds to $t \simeq 10^{12}$. This is an astonishing long time (and incidently supposes that the field stays uniform on the very large corresponding distances).

+oute 4 and 5 illustrates the case $\alpha=.55$. Here the case $B_{0}=10$ is the interesting one. We see 14 revolutions in the $\vec{\xi}$ plane followed by the rather sudden escape of the particle: Again the corresponding time is very large $\theta=8, t=357$ and practical space and time limitations will limit the possibility of seeing these transitions Nevertheless if is interesting to see that the $\vec{\xi} \vec{\eta} \theta$ space with its high degree of compression in space and time is indeed the best suited for description of the motion.

$V$ - THE GROUP OF TRANSFORMATION IN QUANTUM MECHANICS

A) Transformation of the Schroedinger equation

Let suppose one dimensional (to simplify) motion of a particle in a potential $\mathrm{V}(\mathrm{x})$. The description is through the Schroedinger equation

$|40|$

$$
i \frac{\partial \psi}{\partial t}=-\frac{1}{2} \frac{\partial^{2} \psi}{\partial x^{2}}+V(x) \psi=H \psi
$$

we introduce the transformation $\xi=\mathrm{xC}^{-1}$ but dealing with the Schroedinger formalism we cannot yet consider a relation between $\eta$ and $\mathrm{x}$ and $\mathrm{v}$ (we will see later on).

Now we introduce the following transformation on $\psi$

141| $\psi(x, t)=B(t) \exp i \phi \bar{\psi}(\xi, \theta)$

with

$$
\phi=x^{2} K(t)
$$

$$
\theta=\theta(t)
$$

Computing $\partial \psi / \partial t=\partial^{2} \psi / \partial x^{2}$ and introducing these results we obtain for the Schroedinger equation (40). 
|43| $\quad H \psi=\left\{-\frac{1}{2} \frac{B}{C^{2}} \frac{\partial^{2} \bar{\psi}}{\partial \xi^{2}}-\frac{B}{C} \frac{\partial \bar{\psi}}{\partial \xi}(2 i x K)+B \bar{\psi}\left(2 x^{2} K^{2}\right)-i K B \bar{\psi}+V B \bar{\psi}\right\} \exp i \phi$

$|44| \quad i \frac{\partial \psi}{\partial t}=\left\{i B \frac{d \theta}{d t} \frac{\partial \bar{\psi}}{\partial \theta}-\frac{i B}{C^{2}} \times \frac{\partial \bar{\psi}}{\partial \xi} \frac{d C}{d t}-B \bar{\psi} x^{2} \frac{d K}{d t}+i \frac{d B}{d t} \bar{\psi}\right\} \exp i \phi$

the "new Schroedinger equation" we want to write

$|45| \quad-\frac{1}{2} \frac{\partial^{2} \bar{\psi}}{\partial \xi^{2}}+\bar{V} \bar{\psi}=i \frac{\partial \bar{\psi}}{\partial \theta}$

consequently in $(43)=(44)$ we must suppress the temr in $\partial \bar{\psi} / 0 \xi$

$|46| \quad \frac{2 K}{C}=\frac{d c}{d t} \frac{1}{c^{2}}$

moreover equating the first terms in the right hand side of (43)

and (44)

$|47| \quad \frac{d \theta}{d t}=\frac{1}{c^{2}}$

Finally getting rid of the terms in $i \bar{\psi}$

$|48| \quad-K(t)=\frac{1}{B} \frac{d B}{d t}$

Combining (46) (47) and (48) we obtain

$|49| \quad B^{2} C=1$

$|50| \quad K=(1 / 2 c) d c / d t$

while the potential $\bar{V}$ in $(45)$ becomes

$$
\bar{V}=V c^{2}+\left(\frac{d K}{d t}+2 K^{2}\right) x^{2} c^{2}=V c^{2}+\frac{1}{2} c^{3} \frac{d^{2} c}{d t^{2}} \xi^{2}
$$

We see that as in the classical case the new potential is the sum of the rescaled physical potential $\mathrm{V}$ plus a transformation potential. 
If we introduce the fields $\varepsilon=-J \bar{V} / J \xi$ and $E=-J V / J x$ we get

$$
\varepsilon=c^{3} E-c^{3} \frac{d^{2} c}{d t^{2}} \xi
$$

(52) is strictly identical to (14)

B) Introduction of the Wigner distribution function

How can we recuperate the second of equation (31) i.e. the relation between $\eta$ and $v$ and $x$ ? The question is interesting and is answered by considering the Wigner distribution function $|16| f(x, v)$. It must be pointed out that the inysical meaning of this fanction is not clear since this function has not all the good properties characterizing such a distribution (the biggest difficulty being that $f$ can be negative). From a Schroedinger $\psi$ function Wigner defines a distribution in phase space through the relation

$|53| f(x, v)=\frac{1}{2 \pi} \int \psi\left(x+\frac{\Delta}{2}\right) \psi^{*}\left(x-\frac{\Delta}{2}\right) \exp -i v \Delta d \Delta$

from (41)

$$
\psi\left(x+\frac{\Delta}{2}\right)=\bar{\psi}\left(\xi+c^{-1} \frac{\Delta}{2}\right) \quad \exp i K(t)(c \xi+\Delta / 2)^{2} B(t)
$$

$$
\psi^{*}\left(x-\frac{\theta}{2}\right)=\bar{\psi}^{*}\left(\xi-c^{-1} \frac{\Delta}{2}\right) \quad \exp -i K(t)(c \xi-\Delta / 2)^{2} B(t)
$$

We introduce (52) in (51) and use (50)

$$
f(x, v)=\frac{1}{2 \pi} \int B^{2} \bar{\psi}\left(\xi+c^{-1} \frac{\Delta}{2}\right) \bar{\psi}^{*}\left(\xi-c^{-1} \Delta / 2\right) \exp -i \Delta(v-\dot{c} \xi) d \Delta
$$

we introduce $\Delta^{\prime}=C^{-1} \Delta$ and notice (49) that $B^{2} C=1$. We obtain

$|55| f(x, v)=\frac{1}{2 \pi} \int \bar{\psi}\left(\xi+\frac{\Delta^{\prime}}{2}\right) \bar{\psi}^{*}\left(\xi-\frac{\Delta^{\prime}}{2}\right) \exp -i \Delta^{\prime}(c v-\dot{c} \xi) d \Delta^{\prime}$

Defining as in the classical case $\eta=c V-\dot{c} x=c v-\frac{d c}{d t} x ; \xi=c^{-1} x$

$$
|56| \quad f(x, v)=F(\xi, \eta)
$$

and the phase space distribution is invariant as in the classical case. This is indeed the great advantage of the Wigner distribution: 
The classical concepts can be used but of course the Wigner distribution does not obey a classical Vlasov equation.

\section{C) Application to the problem of quantum Harmonic oscillator with varying frequency}

To end this section on quantum transformations let us consider a potential for a one dimentional oscillator given by

$|57| \quad V(x)=\frac{1}{2} \Omega^{2}(t) x^{2}$

the classical problem involves the solution of the equation

$|58| \quad \frac{d^{2} x}{d t^{2}}+\Omega^{2}(t) x=0$

Although the following result has already been established by Lewis and Riesenfeld $|17|$ let us show that if we know a solution of (58) we can solve the quantum case. We transform the equation through a given $C(t)$ and from (57) and (51) we get

$$
\bar{V}=\frac{1}{2} \Omega^{2} C^{4} \xi^{2}+\frac{1}{2} c^{3} \frac{d^{2} C}{d t^{2}} \xi^{2}=\frac{1}{2} c^{3} \xi^{2}\left[\frac{d^{2} C}{d t^{2}}+\Omega^{2}(t) C\right]
$$

If we choose $\ddot{C}+\Omega^{2} C=0, \bar{V}=0$ and the problem is reduced to the problem of free particles. But a difficulty occurs if $C(T)=0$ for $t=T$. $\theta$ goes to infinity and we cannot pass $t=T$. Since another easy to solve problem is the quantum oscillator with a fixed frequency $\Omega^{2}(t)=\omega^{2}$ we can select $C$ as a solution of

$|59| \quad \frac{d^{2} c}{d t^{2}}+\Omega^{2} C=\frac{\omega^{2}}{C^{3}}$

and we have $\bar{V}=\frac{1}{2} \omega^{2} \xi^{2}$, a problem with a known solution. We must simply solve (59). It can be shown that if $\mathrm{C}(0)>0$ the solution of (59) never goes to zero. 
VI - VLASOV POISSON SYSTEM

\section{A) One dimensiona1 problem beam}

We go back to the Vlasov Poisson system. We begin by considering a one dimensional beam problem $B$ with an initial condition as indicated on Fig. 6. It must be pointed out that we have put a limit $V_{0}$ on the modules of the possible velocities. Although physically such an absolute cut off does not exist the number of particle with velocities greater than say $5 \mathrm{~V}_{\mathrm{T}}$ is very small and can be supposed equal to zero. Moreover the beam is limited in space from $x=-L$ to $x=L$ and is homogeneous in this interval. To compute

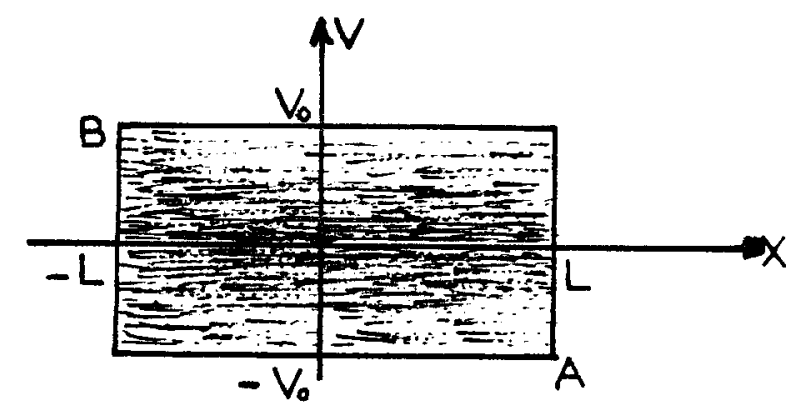

FIG. 6 its evolution we select a $C(t)$ such that $C(0)=1$ and $(d C / d t)=0$ at $(t=0) . \quad$ At initial time $t=\theta=0$ the two phase spaces $\mathrm{x}, \mathrm{v}$ and $\xi, n$ coincides. The Poisson equation (17) can be written

$|60| \quad \frac{\partial \varepsilon}{\partial \xi}=c^{3} \int F(\xi, \eta) d \eta-c^{3} \frac{d^{2} C}{d t^{2}}$ In thezplane at $\theta=0 \quad F(\xi, \eta)=F(\eta)$ for $-L<\xi<L$ and the density $\int \mathrm{F} d \eta=\mathrm{N}$ We select $C$ such that:

$$
C^{3}\left[N-\frac{\partial^{2} C}{\partial t^{2}}\right]=0
$$

with $C(0)=1, \mathrm{dC} / \mathrm{dt}(0)=0$. We get of course

$$
\text { |61| } C=1+\Omega^{2} t^{2} \quad \text { with } \Omega^{2}=N / 2 \text {. }
$$

If we remember that we took $e=m=\varepsilon_{0}=1$ we see that $\Omega^{2}$ is simply the plasma frequency associated to a density half of the initial density of the beam. Now the possibility of selecting such $C(t)$ has two important consequences.

The new field $\mathcal{E}$ is zero and since the beam is uniform it will stays zero. Being homogeneous and with no field acting on it the plasma is on a steady state with no time evolution. 
In fact things are more complex. The steady state character of the beam implies a infinite length of the beam. If boundaries exist, as they do, we must use the concept of contamination already introduced in $|\beta|$. We briefly remind it.

Due to the one dimensional character of the problem supposing moreover a space symmetry, particles outside a limited region do not create any field inside, this property remaining true in cylindrical, and spherical symmetrical structures. As long as these particles do not penetrate physically into this zone they will not influence the behaviour of the particles in this zone. When particles located initially respectively in the inner and outer zones cross a "contamination process" is generated. Obviously the evolution of the contaminated zones is given by the evolution of the two boundaries and more precisely by the (symetric) trajectories of point $A$ and $B$ of Fig. 6. Now the trajectory of point $A$ for example is a simple uniform motion (in the $\xi, n, \theta$ space) with

$|62| \quad \xi_{A}=L-V_{0} \theta$

and the regions $|\xi|<\xi_{A}$ is the uncontaminated zone. The interesting point is that: when $t \rightarrow \infty, \theta \rightarrow$ limit $\theta_{\ell}$ and the contamination will stop if $L>V_{0} \quad \theta_{\ell}$ leaving the central zone $|\xi|<L-V_{0} \theta_{g}$ always uncontaminated. Introducing (31) in (13) we obtain the relation between $\theta$ and $t$

$$
\Omega \theta=\frac{1}{2}\left\{\frac{\Omega t}{1+\Omega^{2} t^{2}}+\operatorname{Arctg} \Omega t\right\}
$$

and when $t \rightarrow \infty, \Omega \theta \rightarrow \pi / 4$. The percentage of uncontaminated particles in Fig. 6 is consequently $1-\pi V_{0} / 4 \Omega L$

Fig. 7 and 8 shows an illustration of this concept. It can be noticed that in the usual phase space we have a simple expansion. Fig. 3 indicates that indeed $\eta$ is an invariant. The computer experiments are of Lagrangian type with plane superparticles, the motion of which is computed through Newtor. and Coulomb 1 aw. 


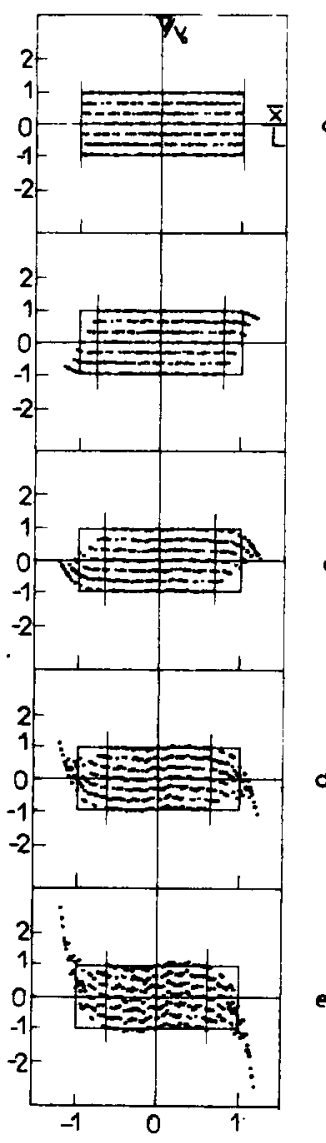

FIG. 7

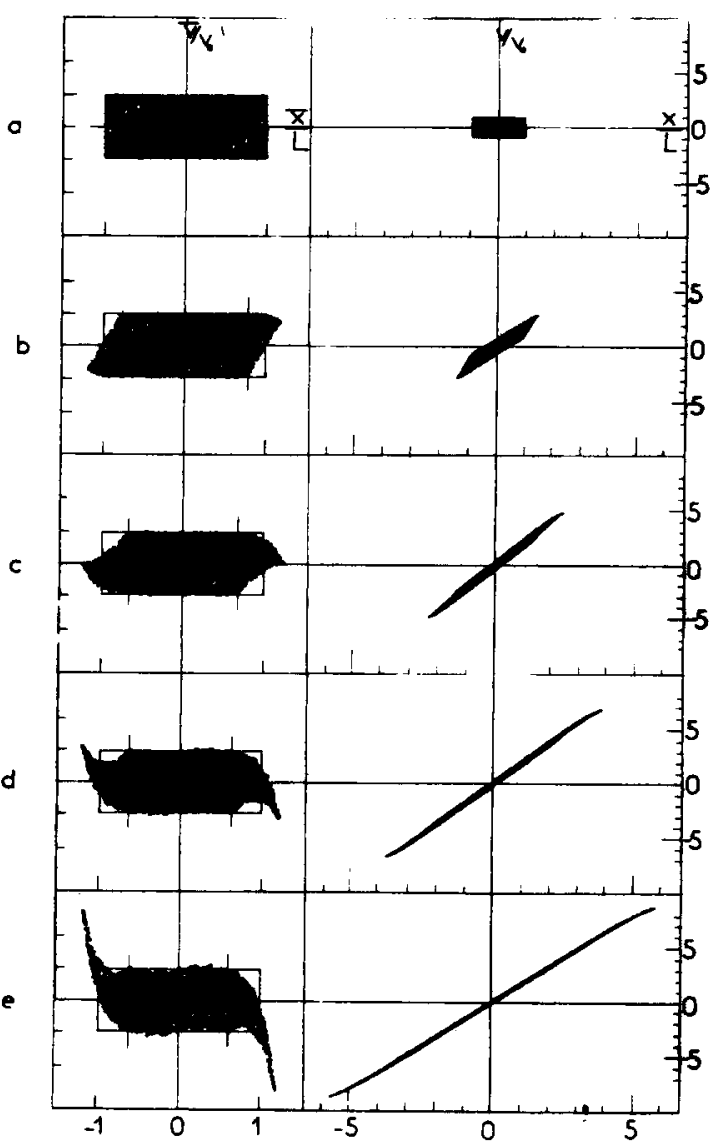

FIG. 8.

Fus 7 -

[Evolution in the phase spaces $x v$ (right) and $x v$ (left) of an horizontal water bag rod. At $t=0$ the cutt off velocities are $\pm V_{0}$ and the rod length is $2 L$. We define $\Omega$ as the plasma frequency corresponding to the density $A V_{0}=1 / 2$ of the density at $t=0$. ( $A$ being the value of the phase space density inside the bag). The problem is entirely characterized by the value of the parameter $L \Omega / V_{0}$ (here equal to 2 ). Figures are respectively given for $\Omega t=a) 0 ; b) 0.5 ; c) 1.0 ; d) 1.5 ; e) 2.0$. The uncontaminated zone is indicated only on the $\overline{x v}$ phase space and is a rectangle delineated by the straight lines $\pm V_{0}$ and the two vertical lines whose abscisses are given by

$$
\pm \bar{x}_{\mathrm{o}} / L=1-V_{0} / 2 \Omega L\left(\tau\left(1+\tau^{2}\right)+\operatorname{Arctg} \tau\right) \text {. }
$$

Here for $\tau \rightarrow \infty$ the percentage of uncontaminated particles go to $1-\tau V_{0} / 4 \Omega L=0.6073$.]

\section{FrG:}

Même diagramme $\overline{x v}$ que pour la figure 1 avec indication de l'évolution de 7 groupes de particules dont les vitesses initiales sont $\pm V_{0}, \pm 2 V_{0} / 3, \pm V_{0} / 3$ et 0 . On voit que dans la zone non contaminée $\bar{v}$ est un invariant. 


\section{B) One dimensional problem-plasma}

For a plasma the corresponding Poisson equation (17)

is written

$|64| \frac{\partial \xi}{\partial \xi}=c^{3} \int F(\xi, \eta) d \eta-c^{4}-c^{3} \frac{d^{2} c}{d t^{2}}$

Starting from a uniform electron plasma $P$ as in Fig. 6

with an initial density $n_{0}$ for the electrons and $N_{0}$ for the motionless ions we select $C(t)$ satisfying $C(0)=\frac{d C}{d t}(0)=0$ and

$|65| \quad n_{0}=C N_{0}+\frac{d^{2} C}{d t^{2}}$

introducing the plasma frequency $\Omega_{p}^{2}=N_{0} e^{2} / m \varepsilon_{0}$ the solution is

$$
C(t)=\frac{n_{0}}{N_{0}}+\left(1-\frac{n_{0}}{N_{0}}\right) \cos \Omega_{p} t
$$

We see two things

$$
\text { If } n_{0} / N_{0}<1 / 2, C(t) \text { will take the value } 0 \text { and for }
$$

this value of the time, $\theta$ will be infinite. We will not be able to compute the behaviour beyond.

$$
\text { If } \mathrm{n}_{0} / \mathrm{N}_{0}>1 / 2, \mathrm{C}(\mathrm{t}) \text { is always positive but does not }
$$

increase with time; when $t \rightarrow \infty, \theta \rightarrow \infty$ and the contamination is always total, irrespective of the system. The time of total contamination is $\mathrm{L} / \mathrm{V}_{\mathrm{o}}$ in the $\theta$ scale. For the uncontaminated zone the density varies as $\mathrm{n}=\mathrm{n}_{\mathrm{o}} \mathrm{C}^{-1}(\mathrm{t})$

$|67| \quad n(t)=\frac{n_{0}}{\left(n_{0} / N_{0}\right)+\left(1-n_{0} / N_{0}\right) \cos \Omega_{p} t}$

The results are very similar to the cold plasma case $|18|$ with density in the centre oscillating at frequency $\Omega$ p. We notice that since $C(t) \rightarrow$ Oimplies $\theta \rightarrow \infty$ we always have total contamination before $n(t)$ blows up in the case $n_{0} / N_{0}<1 / 2$. 


\section{C) One dimensional problem-gravitating gas}

The case of gravitating gas with an initial situation described as in Fig.6 is similar to the B case except a sign in front of $N=\int F d \eta$ and a solution for $C(t)$ given by

[68 $\quad C=1-\Omega^{2} t^{2} \quad$ with $\Omega^{2}=N / 2$

$\Omega^{2}$ is now the Jean's frequency associated to $a$ density equal to half the initial density of the system.

As in the B case the system is stationary in the $\xi$, $\eta$ phase space with

$$
\xi=x\left(1-\Omega^{2} t^{2}\right)^{-1}
$$

$$
\eta=V\left(1-\Omega^{2} t^{2}\right)+2 \Omega^{2} t x
$$

$n$ being an invariant in the uncontaminated zone. Of course the contamination becomes total in a time always smaller than $\Omega^{-1}$ since the relation between $\theta$ and $t$ is now

$$
|70| \quad \Omega \theta=\frac{1}{2}\left(\frac{\Omega t}{1-\Omega^{2} t^{2}}+\operatorname{Arcth} \Omega t\right)
$$

If $2 \mathrm{~L}$ is the length of the system the time of total contamination is given by the solution of the equation $L / V_{0}=\theta$. At time $t$ the density in the incontaminated zone is $N\left(1-\Omega^{2} t^{2}\right)^{-1}$ and for a sufficiently large $N$ a very large increase of the density is consequently possible.Fig. 9 shows the situation for $L \Omega / V_{0}=1$. The invariance of $\eta$ and the shrinking of the uncontaminated zone are well illustrated. 


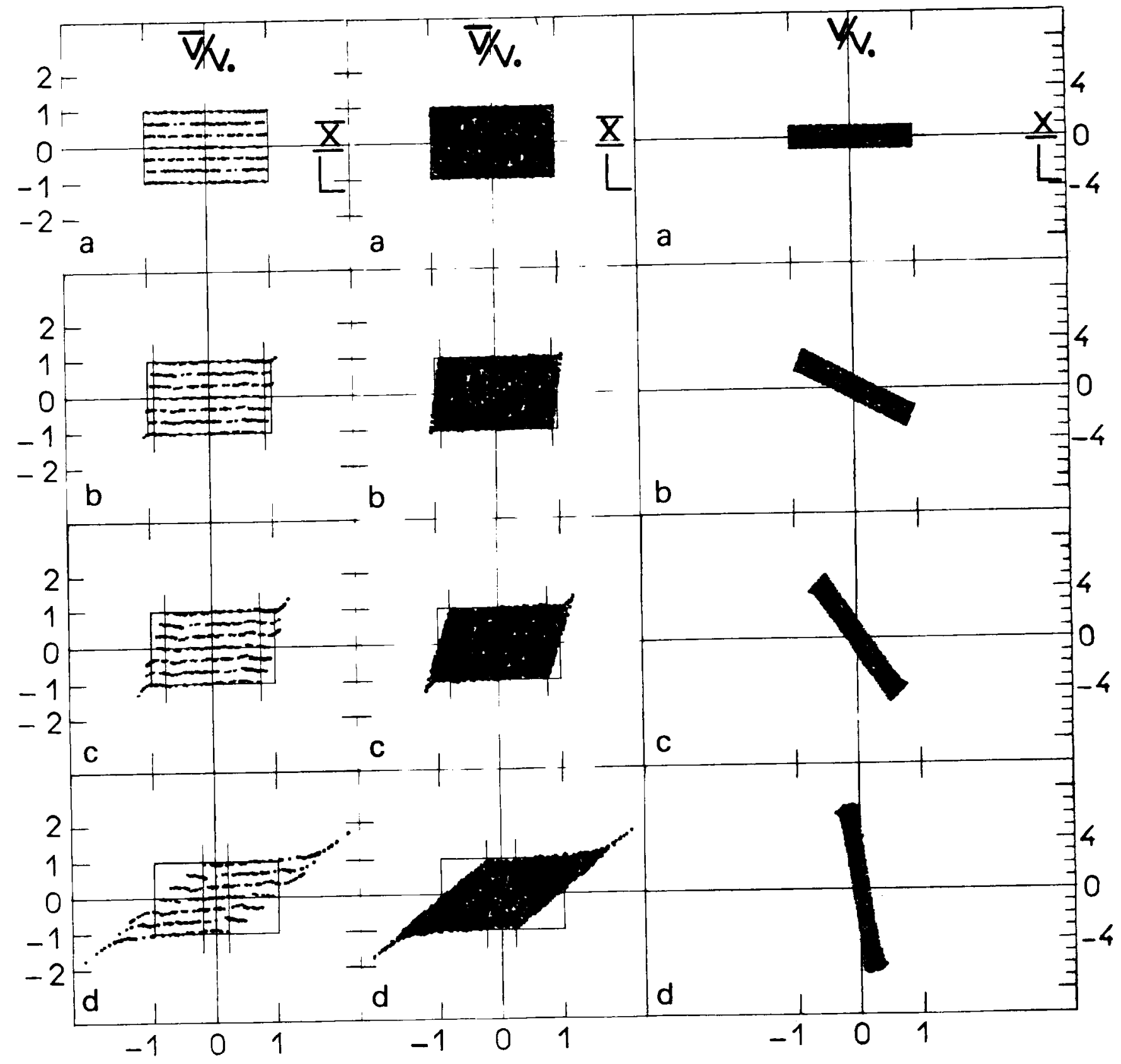

Figure 9 : Evolution in

the phase spaces $x, v$ (right) and $\bar{x}, \bar{v}$ (left) for a limited gravitational horizontal "stick" solution. At initial time the boundaries are $\bar{x}=x= \pm L, \vec{v}=v= \pm v_{0}$. Here the parameter characterizing the problem is $L \Omega / V_{0}=4$. Figures, obtained using 3000 a particle code are respectively given for $\Omega \tau=$ a) 0. ; b) 0.3 ; c) 0.6 ; d) 0.9 . In this case, the collapse and the crossing of all particles in the neighbourhood of the center bring a total contamination for $\Omega \tau=0.92$. It can be seen that, as long as the particles remain uncontaminated, $\vec{v}$ is an invariant. We indicate the evolution of seven groups of particles with initial velocities $\pm v_{0}, \pm 2 / 3 v_{0}, \pm 1 / 3 v_{0}$, and 0 . 
D) Homogeneous systems in cylindrical and spherical geometry (Beam and gravitational gas)

An advantage of our group transformation analysis is that the results can be generalised to cylindrical and spherical geometry altough it must be pointed out that in order to apply the contamination concept we must deal with systems where the particles from outside do not create a field for the particle inside who implies a cylindrical and a spherical geometry in respectively 2 dimensional and 3 dimensional problems. $N$ being the initial density in the $B$ and $G$ cases with $N / 2=\Omega^{2}$ and $n_{0}$ and $N_{0}=\Omega_{p}^{2}$ the electrons and ion density in the $P$ case. We must select $C(t)$ satisfying: in two dimensions (cylindrical):

$$
\begin{aligned}
& \Omega_{p}^{2} C^{2}+2 C \frac{d^{2} C}{d t^{2}}=n_{0} \quad P \text {. } \\
& |71| \quad 2 C \frac{d^{2} c}{d t^{2}}=\Omega^{2} \\
& 2 c \frac{d^{2} c}{d t^{2}}=-\Omega^{2}
\end{aligned}
$$

in three dimensions (spherical)

$$
\begin{array}{lll} 
& \Omega_{p}^{2} C^{3}+3 C^{2} \frac{d^{2} c}{d t^{2}}=n & P \\
32 \mid & 3 c^{2} \frac{d^{2} c}{d t^{2}}=\Omega^{2} & B \\
3 c^{2} \frac{d^{2} c}{d t^{2}}=-\Omega^{2} & G
\end{array}
$$

in all cases $C(t)$ and $d C / d t$ for $t=0$ are respectively 1 and 0

D1) The two dimensional beam problem

In the $2 \mathrm{D} B$ problem the relations between $C, t$ and $\theta$ are given by the following parametric solutions.

$$
\begin{aligned}
& |73| \quad \Omega t=2 \int_{0}^{\sqrt{\log C}} \exp z^{2} d z \\
& |74| \quad \Omega \theta=2 \int_{0}^{\sqrt{\log C}} \exp -z^{2} d z
\end{aligned}
$$


$C(t) \geqslant 1$ and $C(t)$ an always increasing function with time goes to infinity when $t \rightarrow \infty$. $\theta$ goes to a finite limit $\Omega \theta_{\rho}=\sqrt{\pi}$ and the contamination is only partial provided, of course, we can put a limit $V_{0}$ to the velocities and that $R / V_{o}>\theta_{\ell}$. The density in the uncontaminated zone is $\mathrm{NC}^{-2}$ and this gives the law of decrease in the density under the influence of space charge and ballistic effect for a cylindrical beam.

\section{D2) The two dimensional gravitating problem}

In the two dimensional $G$ problem, relations (73) and (74) are just inversed.

$$
\Omega \theta=2 \int_{0}^{\sqrt{-\log c}} \exp z^{2} d z
$$

$$
\Omega t=2 \int_{0}^{\sqrt{-\log c}} \exp -z^{2} d z
$$

$C(t) \leqslant 1$ and $C(t)$ an always decreasing function, with time equal zero for $\Omega t_{c}=\sqrt{\pi}$. Before that time, total contamination has taken places and up to that time we can follow the density increase in the center.

\section{D3) The three dimensional beam problem}

In the3dimensional $B$ problem

Equation (72) B can be easily integrated giving

$$
\begin{aligned}
& |77| \Omega t=\sqrt{\frac{3}{2}}\{\sqrt{c(c-1)}+\operatorname{Arcch} \sqrt{c}\} \\
& |78| \quad \Omega \theta=\sqrt{6} \sqrt{\frac{c-1}{c}}
\end{aligned}
$$

$C(t) \geqslant 1$ is always increasing. $\theta$ goes to the finite limit $\sqrt{6} \Omega^{-1}$ and the contamination is partial. $\mathrm{NC}^{-3}$ gives the evolution of the density in the center of the spherical system. 
D4) The three dimensional gravitational prob1em

In the $3 \mathrm{D} G$ problem, the integration is quite similar to the preceeding one. We get

$|79| \quad \Omega t=\sqrt{\frac{3}{2}}\{\operatorname{Arccos} \sqrt{c}+\sqrt{c(1-c)}$

$|80| \quad \Omega \theta=\sqrt{6} \sqrt{\frac{1-c}{C}}$

$C(t) \leqslant 1$ is always decreasing. Contamination is total in a time always smaller than $\Omega t_{c}=\pi \sqrt{3 / 8}$.

E) Plasma case : two and three dimensions

The plasma case is a little bit more difficult. We must solve (71) P, $d$ being the dimension ( $d=2$ or 3 )

$$
n(t)=n_{0} c^{-d}
$$

Deriving (81) we get $d n / d t$ and $d^{2} n / d t^{2}$

$$
\frac{d^{2} n}{d t^{2}}=\frac{d+1}{d n_{0}} c^{d}\left(\frac{d n}{d t}\right)^{2}-d n_{0} c^{-d-1} \frac{d^{2} c}{d t^{2}}
$$

Introducing $d^{2} C / d t^{2}$ taken from (71) and (72) in (82)

$$
\frac{d^{2} n}{d t^{2}}=\frac{d+1}{d} \frac{1}{n}\left(\frac{d n}{d t}\right)^{2}-n^{2}+\Omega_{p}^{2} n
$$

we write $P=d n / d t$

$$
\frac{d^{2} n}{d t^{2}}=P \frac{d P}{d n}=\frac{d}{d n}\left(P^{2}\right)
$$

Introducing (84) in (83) we obtain the first degree equation $\left(\mathrm{P}^{2}\right.$ function, $\mathrm{n}$ variable)

$$
\frac{d P^{2}}{d n}=2 \frac{d+1}{d} \frac{1}{n} P^{2}-2 n^{2}+2 \Omega_{p}^{2} n
$$


The solution of which being

$$
\text { |86 } \quad p^{2}=K_{0} \pi^{2 \frac{\alpha+1}{\alpha}}-\alpha \Omega_{p}^{2} \pi^{2}-2 \pi^{2 \frac{d+1}{\alpha}} \int \pi^{-2 / d} d n
$$

We must distinguish between $d=2$ and $d=3$

$d=3$ gives no problem in the last integration of (86)

$K_{0}$ is obtained noticing that for $t=0 \quad n=n$. and $d n / d t=P=0$

$|87| \quad K_{0}=3 \Omega_{p}^{2} n_{0}^{-2 / 3}+6 n_{0}^{1 / 3}$

We finally obtain the relation between $t$ and $n$

$$
t=\int_{n_{0}}^{n} x^{-1}\left[3 \Omega_{p}^{2}\left(\left(\frac{x}{n_{0}}\right)^{2 / 3}-1\right)+6 n_{0}\left(\left(\frac{x}{n_{0}}\right)^{2 / 3}-\frac{x}{n_{0}}\right)\right]^{-1 / 2} d x
$$

The denominator of (88) cancels for $n=n_{0}$. It is easy to find the other values for which it is zero. One is negative and without interest, the other is (taking $\Omega_{p}^{2}=K_{0}=1$ )

$|89| \quad x=n_{1}=n_{0}\left[\frac{\sqrt{1+8 R_{0}}+1}{4 n_{0}}\right]^{3}$

If $n_{0}<1, n_{1}>1$ and if $n_{0}>1 \quad n_{1}<1$. Consequently the density is periodic and oscillates between these two values.The period is given by twice the integral (88) taken between the values $\mathrm{n}_{0}$ and $\mathrm{n}_{1}$ (or $\mathrm{n}_{1}$ and $\mathrm{n}_{0}$ ). In contradistinction to the plasma case $n_{0}$ can be as small as we like without appearance of a maximum $\mathrm{n}_{1}$ going to infinity (In the plane case $\mathrm{n}_{1}=\mathrm{n}_{d} /\left(2 \mathrm{n}_{0}-1\right)$. $d=2$ is the last case to be treated. In (86) $\int n^{-2 / d} d n \Rightarrow \log n$. Again we get $K_{0}$ throught $t=0, n=n_{0}$ and $P=0$.

190 $\quad K_{0}=2 \Omega_{p}^{2} n_{0}^{-1}+2 \log n_{0}$ 
$t$ is obtained by integration between $n_{0}$ and $n$

$$
t=\int_{\pi_{0}}^{n} 2^{-1 / 2}\left\{\Omega_{p}^{2} x^{2}\left(\frac{x}{n_{0}}-1\right)-x^{3} \log \frac{x}{n_{0}}\right\}^{-1 / 2} d x
$$

As in the preceeding case the expression between brackets cancels for $x=n_{0}$ and $x=n_{1}$ (one greater, the other smaller than $N_{0}$ ) the density oscillates between these two values and is periodic with the half period given by the integral (91) taken from $n_{0}$ to $n_{1}$ (or $\mathrm{n}_{1}$ to $\mathrm{n}_{0}$ ). As in the 3 dimensional cases no restrictions are put on the value of $n_{0}$, and $n_{0}$ infinity appear. (88) and (91) indicate that in cylindrical and spherical geometry the frequency of oscillation is a function of $n_{0}$ in contradistinction to the result obtained for plane geometry as given by (67) where $\Omega_{p}$ is the oscillation frequency and does not depend of $n_{0}$.

Nevertheless in the linearised limit we recover ' the 3 cases the plasma frequency $\Omega_{p}$. The equation for $c$ can be written

$$
n_{0}=\Omega_{p}^{2} c^{d}+d c^{d-1} \frac{d^{2} c}{d t^{2}}
$$

we suppose that $n_{0}=N_{0}(1+\varepsilon)=\Omega_{p}^{2}(1+\varepsilon)$ and $\varepsilon$ is $\operatorname{smal} 1$ since $n_{0}$ is very close to $N_{0}$. Introducing $\Omega_{p} t=6$ we rewrite (92)

$$
1+\varepsilon=c^{d}+d c^{d-1} \frac{d^{2} c}{d t^{2}}
$$

we write $C=1+\psi$ and linearize neglecting terms in $\psi^{2} \psi^{3} \ldots(92)$ becomes

$$
|94| \quad \varepsilon / d=\psi+\frac{d^{2} \psi}{d \tau^{2}}
$$

The solution of (94) with $\psi=0 \quad d \psi / d \tau=0$ for $\tau=0$ is

$$
i=\varepsilon / d(1-\cos \tau)
$$

Introducing (95) in $\mathrm{n}=\mathrm{N}_{\mathrm{o}}(1+\varepsilon)(1+\psi)^{-\alpha}$ and retalning only the first terms in $\varepsilon$ we get

$$
n=N_{0}(1+\epsilon \cos \sigma)
$$

(96) describes the usual linearised plasma oscillations phenomena. 


\section{APPENDIX}

\section{SELF ADJUSTED TRANSFORMATION}

\section{INTRODUCTION}

We show in this appendix that the time rescaling of formulas (13) is a particular case of a more general theory of self adjusted transformation where we work directly in 4 dimensional space time with the. coordinates and the time expressed as function of a parameter $\lambda$. We indicate that it is possible to find a generalised canonical transformation for $x$ y $z$ and $\lambda$.

\section{CONFIGURATION SPACE $\mathbb{C}$}

Let $q=\left\{q^{i}\right\}$ the generalized coordinates $\left(q^{i}=x, y, z, t\right)$ they are independant variables in $C$ space $C$ is then the set of all the points $M$ of coordinates $q$. Let us now have a relation ship between the $q^{i}$. This is the same as to give a parameter $\lambda$, the variation of which describes a curve in $C$. Thus each point $M$ can be said to belong to a curve $\Gamma$ of $\mathbb{C}$, the position of $M$ or $\Gamma$ is given by $\lambda$, and the curve $\Gamma$ itself depends on the initial position of $M$.

A tangent vector along the curve $\Gamma$ is $u^{i}=\left.\frac{d q^{i}}{d \lambda}\right|_{\lambda=\lambda_{0}}$

Thus : give a set of curves (i.e. a parametrization, i.e. trajectories), and get a tangent space.

The tangent base is $\frac{\partial}{\partial q^{i}}$.

Any tangent vector can read then $x=\xi^{i} \frac{\partial}{\partial q^{i}}$

The Gual tangent base is $\hat{d} q^{i}$

$\hat{d} q^{i}$ is the usual definition of exte rior differential. 
$-32-$

The exterior derivative of a scalar function $f$, along a vector field u is defined as :

$$
\langle\hat{d} f, u\rangle=u^{i} \partial_{i} f
$$

$$
\text { then if } \begin{aligned}
& f=q^{i} \text { and } u=\frac{\partial}{\partial q^{j}} \\
&\left\langle\hat{d} q^{i}, \frac{\partial}{\partial q^{j}}\right\rangle=\frac{\partial q^{i}}{\partial q^{j}}=\delta_{j}^{i}
\end{aligned}
$$




\section{PHASE SPACE $\mathbb{P}$}

According to Lagrangian formulation mechanics in Space time, trajectories depend upon integration of 4 second order partial differential equation, thus depend on 8 parameters.

Therefore we are bound to work in an 8 dimensional space (space and there coordinates, and momentum and Energy coordinates) Mechanical lows in $\mathbb{P}$ space are then given by

- the knowledge of the Hamiltonian function $H(p, q)$

- the application of a variational principe on the

$$
1 \text { form } \quad \omega=p_{i} \hat{d} q i-H(p, q) \hat{d} \lambda
$$

this gives us the trajectories in phase space

$$
\begin{aligned}
& \frac{d p_{i}}{d \lambda}=-\frac{\partial H}{\partial q^{i}} \\
& \frac{d q^{i}}{d \lambda}=\frac{\partial H}{\partial p_{i}}
\end{aligned}
$$

which is nothing else than Hamilton's equations

\section{GENERALIZED CANONICAL TRANSFORMATION}

We know that Hamilton's equations are invariant under any canonical transformation which means that if we go over to new coordinates $\bar{q}^{i}(q, p)$ and new momenta $\bar{p}_{i}(q, p)$ in such a way as to have a canonical transformation, then

$$
\frac{d \bar{p}_{i}}{d \lambda}=-\frac{\partial \bar{H}}{\partial \bar{q}} i \text { and } \quad \frac{d \bar{q}^{i}}{d \lambda}=+\frac{\partial H}{\partial \bar{p}_{i}}
$$

and we see that the parameter is still $\lambda$.

We are now interested in transformations which are canonical in coordinates and moments but which also change the parameter to a new one, $\bar{\lambda}$. 
Therefore the transformation is defined by

$$
\frac{d \bar{\lambda}}{d \lambda}=\frac{1}{\alpha^{2}(\lambda)} \quad \begin{aligned}
& \text { relationship between } \bar{\lambda} \text { and } \lambda \\
& \text { (renormalized parametrization) }
\end{aligned}
$$

and by

$$
\Phi(q, \bar{p}, \lambda) \text { generating function. }
$$

We restrict ourselves now to transformations $\bar{q}^{i}(q, \lambda)$ where the new coordinates do not depend on the momenta. The transformation is then

$$
\begin{array}{ll}
\frac{d \bar{\lambda}}{d \lambda}=\frac{1}{\alpha^{2}(\lambda)} & \text { change of parameter } \\
\bar{q}^{i}=g^{i}(q, \lambda) & \text { change of coordinate } \\
\Phi=\bar{p}_{l} g^{i}(q, \lambda) & \text { generating function }
\end{array}
$$

If we write then $\quad A_{i}^{j}(q, \lambda)=\frac{\partial q^{j}}{\partial q^{i}}$ and $B=A^{-1}$

Then the new momenta are given by

$$
P_{i}=A_{i}^{j} \bar{P}_{j} \quad \text { or } \quad \bar{P}_{i}=B_{i}^{j} P_{j}
$$

and the new Hamiltonian is $\bar{H}(\bar{p}, \bar{q})=\alpha^{2}\left(H+\frac{\partial \Phi}{\partial \lambda}\right)$

The tangent space is generated by the Jacobian

$$
\frac{D(\bar{\lambda}, \bar{q}, \bar{p})}{D(\lambda, q, p)}=\left[\begin{array}{ccc}
\frac{1}{\alpha^{2}} & \frac{\partial q^{i}}{\partial \lambda} & p_{j} \frac{\partial B_{i}^{j}}{\partial \lambda} \\
0 & A_{j}^{i} & p_{k} \frac{\partial B_{j}^{k}}{\partial q_{i}} \\
0 & 0 & B_{j}^{i}
\end{array}\right]
$$

then if we define

$$
\begin{array}{ll}
{[q, H]^{i}=\frac{d q^{i}}{d \lambda}-\frac{\partial H}{\partial p_{i}}} & {\left[p, H \rrbracket_{i}=\frac{d p_{i}}{d \lambda}+\frac{\partial H}{\partial q^{i}}\right.} \\
{[\bar{q}, \bar{H}]^{i}=\frac{d \bar{q}^{i}}{d \bar{\lambda}}-\frac{\partial \bar{H}}{\partial \overline{p_{i}}}} & {[\bar{p}, \bar{H}]_{i}=\frac{d \overline{p_{i}}}{d \bar{\lambda}}+\frac{\partial \bar{H}}{\partial \bar{q}^{i}}}
\end{array}
$$


$-35-$

note that in $(q, p)$ space, directional derivative is defined by the $\lambda$ parameter, while in $(q, p)$ space, it is defined by the parameter $\bar{\lambda}$.

Straight forward calculations give

$$
\begin{aligned}
& \left.[q, H]^{i}=\frac{B_{j}^{i}}{\alpha^{2}} \llbracket \bar{q}, \bar{H}\right]^{j} \\
& {\left[P, H \rrbracket_{i}=\frac{A_{i}^{j}}{\alpha^{2}}\left[\bar{P}, \bar{H} \rrbracket_{j}-\frac{P_{k}}{\alpha^{2}} \frac{\partial B_{j}^{k}}{\partial q^{i}}\left[\bar{q}, \bar{H} \rrbracket^{j}\right.\right.\right.}
\end{aligned}
$$

If Hamilton's equations hold in $q(\lambda), p(\lambda)$ space, $\llbracket q, H]^{i}=0$ and $\llbracket P, H \rrbracket=0$, they hold too in $\bar{q}(\bar{\lambda}), \bar{p}(\bar{\lambda})$ space. But levidently the trajectories do not correspond in the two spaces.

4. APPLICATION TO PLASMA PHYSICS

We choose the following generating function

$$
\Phi=\bar{P}_{t} g(t, \lambda)+\bar{r}_{i} \frac{x^{i}}{\alpha(\lambda)}
$$

where $p_{t}$ will be the momentum, canonical conjugate of the time variable $t$, this is the energy.

$$
\begin{aligned}
& \pi_{i} \text { are spatial momenta } \\
& x^{i} \text { are the spatial coordinates }
\end{aligned}
$$

We have then

$$
\bar{q}^{i}=\frac{\partial \Phi}{\partial \bar{P}_{i}} \quad ; \quad P_{i}=\frac{\partial \Phi}{\partial q^{i}} ; \bar{H}=\alpha^{2}\left(H+\frac{\partial \Phi}{\partial \lambda}\right)
$$

Therefore

$$
\bar{t}=g(t, \lambda) ; \bar{x}^{i}=\frac{x^{i}}{\alpha(\lambda)} ; \bar{l}_{i}=\frac{\bar{\pi}_{i}}{\alpha(\lambda)}
$$

we use now, along a trajectory, $t$ as the parameter, thus $\lambda=t$ and we like to have, on the transformed curve $\bar{\lambda}=\bar{t}$. Therefore, when we derive $\bar{t}=g(t, \lambda)$ we have

$$
\frac{d \bar{t}}{d t}=\frac{d \bar{t}}{d \bar{\lambda}} \cdot \frac{1}{\alpha^{2}}=\frac{\partial g}{\partial t} \cdot \frac{d t}{d \lambda}+\frac{\partial g}{\partial \lambda}=\frac{\partial g}{\partial t}+\frac{\partial g}{\partial \lambda}=\frac{1}{\alpha^{2}}
$$


we obtain

$$
\frac{\partial g}{\partial t}+\frac{\partial g}{\partial \lambda}=\frac{1}{\alpha^{2}}
$$

this is a $1^{\text {st }}$ order partiel differential equation, which we can integrate

$$
g=F(t-\lambda)+\int_{a}^{\lambda} \frac{d \sigma}{\alpha^{2}(\sigma)}
$$

for $\lambda=t$ and for a judicious choice of constant "a" we have :

$$
E=\int_{0}^{t} \frac{d \sigma}{\alpha^{2}(\sigma)}
$$

if we now consider Vlasov's equation:

$$
\frac{\partial f}{\partial t}+\vec{v} \cdot \frac{\partial f}{\partial \vec{x}}=(\vec{E}+\vec{v} \wedge \vec{B}) \cdot \frac{\partial f}{\partial \vec{v}}
$$

Maxwe11's equations:

$$
\begin{aligned}
& \text { curl } \vec{E}=-\frac{\partial \vec{B}}{\partial t} ; \operatorname{div} \vec{B}=0 \\
& \text { curl } \vec{B}=\mu_{0}\left(\frac{\partial \vec{E}}{\partial t}-\int \vec{v} f d^{3} \vec{v}\right) ; \operatorname{div} \vec{E}=\delta-\int d d^{3} \vec{v}
\end{aligned}
$$

with initial condition, ( $\delta=$ cte.)

$$
f(\vec{x}, \vec{v}, 0)=g(\vec{x}, \vec{v})
$$

and with normalization relation

$$
n_{0}=\int f d^{3} \vec{v} d^{3} \vec{x}
$$

Let us make the transformation :

$$
\begin{aligned}
& \theta=\int_{0}^{t} \frac{d \sigma}{\alpha^{2}(\sigma)} ; D(\theta)=\frac{1}{\alpha(t)} \\
& \vec{\xi}=D(\theta) \vec{x} ; \vec{\eta}=\frac{\vec{v}}{D(\theta)}+\dot{D}(\theta) \vec{x} \\
& f(\vec{x}, \vec{v}, t)=F(\theta, \vec{\xi}, \vec{\eta})
\end{aligned}
$$

as we have seen in paragraphe (3), Hamilton's equations in the new coordinates give a new Lorentz force

$$
\frac{d \vec{\eta}}{d \theta}=\vec{\varepsilon}+\vec{\eta} \wedge \vec{\gamma}
$$

this results directly from the variation of the action $S(\vec{x}, t)$ which becomes $\varphi(\vec{\xi}, \theta)$ in the new space and is related by

$$
S(\vec{x}, t)=\varphi(\vec{\xi}, \theta)
$$


The transformation law for the Halmitonian function can be written :

$$
D(\theta) \cdot \bar{H}(\vec{\xi}, \vec{\eta}, \theta)=\alpha(t) \cdot H(\vec{x}, \vec{v}, t)+\alpha(t) \cdot \frac{\partial \Phi}{\partial t}
$$

We see that the energy is not conserved in the transformation and if $\mathrm{H}$ doesmot depend on $t, \overline{\mathrm{H}}$ do depend on $\theta$. This peculiarity is due to the fact that we are dealing now with a new time parameter $\theta$ which is not uniform.

We are able now to see that if the new electromagnetic field is defined as

$$
\begin{aligned}
& \vec{B}=D^{2}(\theta) \cdot \overrightarrow{\mathcal{H}}(\theta, \vec{\xi}) \\
& \vec{E}=D^{3}(\theta) \cdot \vec{\varepsilon}(\theta, \vec{\xi})+\ddot{D}(\theta) D(\theta) \vec{\xi}+\dot{D}(\theta) D^{2}(\theta) \vec{\xi} \wedge \overrightarrow{\mathcal{H}}(\theta, \vec{\xi})
\end{aligned}
$$

(new field is $\vec{\varepsilon}, \overrightarrow{\mathcal{C}}$ )

Then Vlasov's equation is the same in the new variables.

$$
\frac{\partial F}{\partial \theta}+\vec{\eta} \cdot \frac{\partial F}{\partial \vec{\xi}}=(\vec{\varepsilon}+\vec{\eta} \wedge \overrightarrow{\mathcal{H}}) \cdot \frac{\partial F}{\partial \vec{\eta}}
$$

The new electromagnetic field does not depend on $\eta$, which was to be expected, since we like to have in the new variables a field depending only on the variables $(\theta, \vec{\xi})$.

Clearly, two of Maxwell's equations are also immediately satisfied :

$$
\begin{aligned}
& \operatorname{div} \vec{B}=D^{2} \operatorname{Div} \overrightarrow{\mathcal{C}} \cdot D=0 \Rightarrow \operatorname{Div} \cdot \overrightarrow{\mathcal{C}}=0 \\
& \text { curl } \vec{E}=-\frac{\partial \vec{B}}{\partial t} \Rightarrow \text { Gurl } \vec{\varepsilon}=-\frac{\partial \vec{H}}{\partial \theta}
\end{aligned}
$$

The charge density becomes

$$
\int f d^{3} \vec{v}=D^{3} \int F d^{3} \vec{\eta}
$$

and the current density becomes

$$
\int \vec{v} f d^{3} \vec{v}=D^{4} \int \vec{\eta} F d^{3} \vec{\eta}-\dot{D} D^{3} \vec{\xi} \int F d^{3} \vec{\eta}
$$

The normalizing condition is also conserved, since the transformation is canonical and lets volume element of phase space invariant.

$$
n_{0}=\int f d^{3} \vec{v} d^{3} \vec{x} \Longrightarrow n_{0}=\int F d^{3} \vec{\eta} d^{3} \vec{\xi}
$$




\section{CONCLUSION}

We have seen in the body of the paper that the time rescaling was an interesting concept allowing to predict, anatytically or numerically, certain asymptotic properties of a physical system. Usually we work in coordinate-momentum space and the time $t$ is considered as the parameter, coordinates and momentum being expressed as function of $t$. Nevertheless, in some situations (especially in problems involving general relativity) it is simple to consider the time as a coordinate and to express everything as a function of a new parameter (proper time). We have shown that a rescaling of this parameter leaving invariant the canonical transformation formalism is also possible.

\section{ACKNOWLEDGEMENTS}

For fruitful discussions and cooperation on the numerical simulation work the authors wish to thak Dr. M. NAVET.

We would like also to thank Miss F. FOURNIER for the ardous and meticulous typing of the manuscript.

Supported by Instituto de Estudios Nucleares (J.E.N.).SPAIN 


\section{BIBL IOGRAPHY}

11 M.R. FEIX, Some problems and methods in computational plasma physics. Proceedings of the Culham S.R.C. Symposium on turbulence and non linear effects in Plasma

B.E. Keen and E.W. Laing Editors, pages 139-183.

|2| M.R. FEIX, Crossfertilization between Plasma, Stellar Dynamics and Hydrodynamics, Dynamics of stellar systems A. Hay1i Ed., Reidel 1975, pages 179-194.

|31 J. BITOUN, A. NADEAU, J. GUYARD, M.R. FEIX, Jour . of Comp. Physics 12, 315-330, 1973.

14| A. NADEAU, J.P. VEYRIER, M.R. FEIX, Numerical and Analytical alternative to the Krylov Bogoliubov method. Applications to slightly non linear autonomous and monautonomous systems. 2nd European Conference on Comp. Physics. D. Biskampf Ed. Munich April 76.

|5| BERNSTEIN I.B., GREENE J.M. and KRUSHEL M.D., Phys . Rev. 108, 5461957.

|61 MORSE R.L. and NIELSON C.W., Phys of Fluids 12, 2418, 1969.

|7| HSUAN H.C.S., LONNGREN K.E. and AMES W.F., Journal of Engineering Mathematics Vo1 8, 303, 1974.

|8| SHEN H. and AMES W.F., Phys. Letters 49A, 313, 1974.

|9| ZABUSKY N.J. and KRUSKAL M.D., Physical Review Letters 15, 240,1965 .

$|10|$ COURANT E.D. and SNYDER H.S., Annals of Phys. 3, 1, 1958. 
|11) LEWIS H.R., Jour. of Math. Phys., 9 1976, 1968.

|12| GUYARD J, NADEAU A., BAUMANN G., FEIX M.R., Jour. Maths. Phys. 12, 488, 1971 .

|13| BURGAN J.R., GUTIERREZ J., FIJALKOW E., NAVET M., FEIX M.R., Journa1 de Physique lettres 38, L 161 1977.

$|14|$ DIRAC P.A.M. Proc. Roy. Society, A 333, 403, 1973.

|15| DIRAC P.A.M., Proc. Roy, Society, A 155, 199, 1938.

|16| J.E. MOYAL, Proceedings Cambride Philosophical Society, 45, 99, 1949.

|17| LEWIS H.R. and RIESENFELD W.B., Jour. Maths. Phys. 10, $1458,1968$.

|18| G. KALMAN, Anan. Phys 10, 1, 1960.

$|19|$ V.B. BARANOV, Sov. Phys. Tech. Phys. 21, 720, 1976

|20| BURGAN J.R., GUTIERREZ J., FIJALKOW E., NAVET M., FEIX M.R., "Self Similar solutions for Vlasov and Water Bag Models". To be pub1ished. 


\section{CRPE}

Centre de Recherches en Physique de l'Environnement terrestre et planétaire

Avenue de la Recherche scientifique 45045 ORLEANS CEDEX
Département PCE Physique et Chimie de l'Environnement

Avenue de la Recherche scientifique 45045 ORLEANS CEDEX
Département ETE Etudes par Télédétection de l'Environnement

CNET - 38-40 rue du général Lecle 92131 ISSY-LES-MOULINEAUX 